\title{
Dual expression of Atoh1 and Ikzf2 promotes transformation of adult cochlear supporting cells into outer hair cells
}

\author{
Suhong Sun ${ }^{1,2 \dagger}$, Shuting $\mathrm{Li}^{1,2 \dagger}$, Zhengnan Luo ${ }^{1,2 \dagger}$, Minhui Ren ${ }^{1,2}$, Shunji He', \\ Guangqin Wang ${ }^{1,2}$, Zhiyong Liu ${ }^{1,3 *}$
}

${ }^{1}$ Institute of Neuroscience, State Key Laboratory of Neuroscience, CAS Center for Excellence in Brain Science and Intelligence Technology, Chinese Academy of Sciences, Shanghai, China; ${ }^{2}$ University of Chinese Academy of Sciences, Beijing, China; ${ }^{3}$ Shanghai Center for Brain Science and Brain-Inspired Intelligence Technology, Shanghai, China

*For correspondence: Zhiyongliu@ion.ac.cn

'These authors contributed equally to this work

Competing interest: See page 24

Funding: See page 24

Received: 14 January 2021

Preprinted: 21 January 2021

Accepted: 02 September 2021

Published: 03 September 2021

Reviewing Editor: Doris $\mathrm{K} W \mathrm{u}$, NIDCD, NIH, United States

(c) Copyright Sun et al. This article is distributed under the terms of the Creative Commons Attribution License, which permits unrestricted use and redistribution provided that the original author and source are credited.

\begin{abstract}
Mammalian cochlear outer hair cells $(\mathrm{OHCs})$ are essential for hearing. Severe hearing impairment follows $\mathrm{OHC}$ degeneration. Previous attempts at regenerating new $\mathrm{OHCs}$ from cochlear supporting cells (SCs) have been unsuccessful, notably lacking expression of the key $\mathrm{OHC}$ motor protein, Prestin. Thus, regeneration of Prestin+ $\mathrm{OHCs}$ represents a barrier to restore auditory function in vivo. Here, we reported the successful in vivo conversion of adult mouse cochlear SCs into Prestin+ $\mathrm{OHC}$-like cells through the concurrent induction of two key transcriptional factors known to be necessary for OHC development: Atoh1 and Ikzf2. Single-cell RNA sequencing revealed the upregulation of $729 \mathrm{OHC}$ genes and downregulation of $331 \mathrm{SC}$ genes in OHC-like cells. The resulting differentiation status of these $\mathrm{OHC}$-like cells was much more advanced than previously achieved. This study thus established an efficient approach to induce the regeneration of Prestin+ $\mathrm{OHCs}$, paving the way for in vivo cochlear repair via SC transdifferentiation.
\end{abstract}

\section{Introduction}

Hair cells (HCs) are the auditory sensors that enable vertebrates to hear. These cells are located in the auditory epithelium, the latter referred to as the organ of Corti (OC) (Wu and Kelley, 2012). Located near $\mathrm{HCs}$ are several supporting cell (SC) subtypes, which, from the medial side to lateral side, are Pillar cells (PCs) and Deiters' cells (DCs) (Montcouquiol and Kelley, 2020). Auditory HCs comprise two subtypes, inner and outer $\mathrm{HCs}$ (IHCs and OHCs). IHCs are the primary sensory cells and innervated by type-I cochlear spiral ganglion neurons (SGNs). IHCs specifically express vGlut3, encoded by Slc17a8, which is required for sound information transmission from IHCs to SGNs (Li et alo, 2018b); consequently, Slc17a $8^{-/}$mice are completely deaf (Ruel et al., 2008; Seal et al., 2008). In contrast, $\mathrm{OHCs}$ act as sound amplifiers and lack vGlut3. OHCs express a motor protein unique to them, Prestin, encoded by Slc26a5 (Zheng et al., 2000). Prestin mediates electromotility, which enables OHCs to function as sound amplifiers, an important step in broadening our ability to perceive sounds. Indeed, Prestin-deficient $\left(\mathrm{Slc26a5^{-1 }}\right.$ ) mice show severe hearing impairment (Liberman et al., 2002). IHCs and OHCs are believed to originate from communal pool of Atoh1+ progenitor cells (Driver et al., 2013; Groves et al., 2013; Matei et al., 2005; Tateya et al., 2019).

Atoh1 is a bHLH transcriptional factor (TF) that is necessary for specifying general $\mathrm{HC}$ fate, and, accordingly, both $\mathrm{IHCs}$ and $\mathrm{OHCs}$ are lost in Atoh $1^{-1-}$ mice (Bermingham et al., 1999). Two additional 
$\mathrm{TFs}$, encoded by Insm 1 and Ikzf2, are known to be required for specifying $\mathrm{OHC}$ fate and/or repressing IHC fate (Chessum et al., 2018; Wiwatpanit et al., 2018). Furthermore, OHCs in Insm $1^{-1-}$ and Ikzf2 point-mutant mice tend to transdifferentiate into IHCs. Insm 1 and Ikzf2 differ in that the former is only transiently expressed in differentiating OHCs (Lorenzen et al., 2015), whereas the latter is expressed permanently throughout the lifetime of OHCs (Chessum et alı, 2018). Unlike IHCs, OHCs are highly vulnerable to ototoxic drugs, noise, and aging and their damage is a common cause of hearing impairment. Non-mammalian vertebrates such as fish and chicken are able to regenerate $\mathrm{HCs}$ from neighboring SCs, during which key HC developmental genes (e.g. Atoh1) are reactivated (Atkinson et alı, 2015). Mammals have lost this regenerative capacity (Janesick and Heller, 2019). Nonetheless, recent single-cell transcriptomic analyses of cochlear cells suggest that some SCs share recent progenitors with OHCs (particularly Lgr5+ PCs and DCs populations) and are thus regarded as a promising source for new OHCs (Chai et al., 2012; Kolla et al., 2020; McLean et al., 2017). Importantly, PCs and DCs are also in close physical proximity to $\mathrm{OHCs}$ requiring minimal if any displacement.

In previous work, we demonstrated that in vivo ectopic Atoh1 expression in neonatal and juvenile SCs (primarily PCs and DCs) is sufficient to convert these cells into nascent $\mathrm{HCs}$ that express early HC markers such as Myo6 and Myo7a (Liu et al., 2012a). In contrast, adult PCs and DCs are not sensitive to ectopic Atoh1 expression (Kelly et alo, 2012; Liu et alı, 2012a), unless additional manipulations are performed (Walters et al., 2017). Despite this progress, no newly in vivo transformed HCs has been reported to express Prestin (Chai et al., 2012; Liu et al., 2012a; Walters et al., 2017). Thus, an outstanding challenge is how to regenerate Prestin+ OHCs from SCs, particularly from adult lesioned cochlea; indeed, $\mathrm{OHC}$ damage is the much more prevalent in the elderly population, than in younger generations. As ectopic lkzf2 induction in IHCs leads to ectopic Prestin expression (Chessum et al,, 2018), we hypothesized that together Atoh1 and Ikzf2 might synergistically reprogram adult PCs and $\mathrm{DCs}$ into not only HCs, but into further differentiated Prestin+ OHCs.

To test this hypothesis, we generated genetic mice lines that allowed us to conditionally and concurrently induce ectopic expression of Atoh1 and Ikzf2 in adult cochlear SCs (primarily PCs and $D C s)$, with or without pre-damaging wild-type OHCs. In summary, the results that emanated from our comprehensive genetic, transcriptomic, immunostaining, morphological, and fate-mapping analyses supported our general hypothesis. Indeed, new Prestin+ $\mathrm{OHC}$-like cells were frequently observed across the entire cochlear duct; interestingly, this occurred much more readily when endogenous $\mathrm{OHCs}$ were pre-damaged. To the best of our knowledge, this is the first report of in vivo generation of Prestin+ OHC -like cells from adult cochlear SCs. Our findings identify Atoh1 and Ikzf2 as potential targets for $\mathrm{OHC}$ regeneration therapy in hearing-impaired patients. Nonetheless, future work is still called for as the Prestin+ $\mathrm{OHC}$-like cells were still insufficiently differentiated to restore hearing loss caused by $\mathrm{OHC}$ damage.

\section{Results}

\section{Generation of conditional Ikzf2-overexpression mouse model}

To test whether concurrent Atoh1 and Ikzf2 expression was sufficient to induce transdifferentiation of adult SCs into $\mathrm{OHCs}$, we needed various conditional transgenic lines. A conditional Atoh1overexpression transgenic strain, CAG-Loxp-stop-Loxp-Atoh1*2xHA+ (CAG-LSL-Atoh1+ for short) already existed, and its ability to efficiently drive high Atoh 1 protein expression was previously confirmed (Liu et alo, 2012a). In this mouse line, 2x HA fragments were tagged to the C-terminus of Atoh1 as a practical means for reading out expression. However, a similar conditional expression model for Ikzf2 was not available. Therefore, we began by generating a Rosa26-CAG-Loxp-stop-

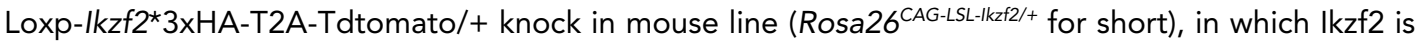
similarly tagged with $3 \times \mathrm{HA}$ fragments at its $\mathrm{C}$-terminus (Figure 1-figure supplement $1 \mathrm{~A}-\mathrm{C}$ ). The mouse line was generated following the CRISPR/Cas9 gene-targeting approach (Li et alo, 2020b), Southern blotting results confirmed the lack of random donor DNA insertion into the host mouse genome (Figure 1-figure supplement 1D,E), and PCR-genotyping of tail DNA allowed us to readily distinguish among wild-type, heterozygous, and homozygous littermates (Figure 1-figure supplement 1F). Both heterozygous and homozygous Rosa26 $6^{\text {CAG-LSL-kf2/+ }}$ mice were healthy and fertile and did not display any noticeable abnormalities. 
The genetic constructs were designed such that upon Cre-mediated recombination, Tdtomato and Ikzf2 would be translated, initially together from the same polycistronic mRNA, and subsequently cleaved by the 2A peptide (Li et al., 2020a); and once triggered, expression of Ikzf2 and Tdtomato would be permanent, enabling genetic fate-mapping analysis at single-cell resolution. Thus, this construct, when used in conjunction with a SC-specific Cre-driver, would readily allow us to distinguish potential new OHCs (Tdtomato+) derived from adult cochlear SCs (primarily PCs and DCs in this study) from endogenous $\mathrm{OHCs}$ (Tdtomato-). Due to the lack of high-quality commercial antibodies against either Atoh1 or Ikzf2, we used anti-HA antibody to assess the presence of ectopic Atoh1 and Ikzf2 proteins in CAG-LSL-Atoh1+ and Rosa26 ${ }^{\text {CAG-LLL-kzz2/+ }}$ strains, respectively.

\section{Induction of ectopic lkzf2 in IHCs is sufficient to induce OHC-markers, prestin, and Ocm expression, as well as reduce IHC-marker, Slc7a14 expression}

We began by validating the ability of the new mouse model (Rosa26 ${ }^{\text {CAG-LSL-kfz2/+ })}$ to drive ectopic and 'functional' Ikzf2 expression. It is known that IHCs, infected with an Anc80-virus expressing Ikzf2 (Chessum et al., 2018), begin synthetizing the typically OHC-exclusive protein, Prestin (Fang et al., 2012; Liberman et al., 2002; Zheng et al., 2000). We therefore used ectopic Prestin expression as a proxy for 'functional' Ikzf2 expression in IHCs. More specifically, we crossed the transgenic Atoh1CreER+ mouse line, in which Cre activity is restricted to HCs (both IHCs and OHCs) (Chow et al.,

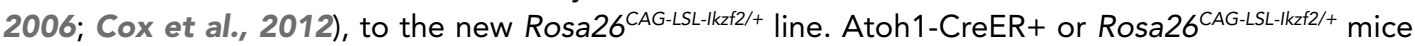
were used as controls, and Atoh1-CreER+; Rosa26 $6^{\text {CAG-LSL-kkf2/+ }}$ mice were experimental group mice. All groups were administered tamoxifen at postnatal day 0 (P0) and P1, and sacrificed and analyzed at P42 (Figure 1A, Figure 1-figure supplements 2 and 3).

We focused our analysis on the IHC Prestin, HA (Ikzf2), and Tdtomato expression patterns, but we did note that $\mathrm{OHCs}$ were also directly affected by the manipulation. In the Atoh1-CreER+ control group $(n=3)$, neither Prestin nor Tdtomato was expressed in IHCs at P42 (Figure 1B-B"). In contrast, in the experimental P42 mice $(n=3)$ Prestin was ectopically expressed in IHCs that were vGlut3+/ Tdtomato+ (arrows in Figure $1 \mathrm{C}^{-\mathrm{C}^{\prime \prime}}$ ) and, as expected, not in IHCs that were vGlut3+/Tdtomato(asterisks in Figure $1 \mathrm{C}-\mathrm{C}^{\prime \prime \prime}$ ). All Tdtomato+ $\mathrm{IHCs}$ were Prestin+ and all Prestin+ IHCs were Tdtomato+. Quantification revealed that $30.8 \% \pm 4.2 \%, 38.4 \% \pm 1.6 \%$, and $70.7 \% \pm 6.0 \%$ of $\mathrm{IHCs}$ were Prestin+ in basal, middle, and apical turns, respectively, at P42 (Figure 1D, Figure 1-figure supplement 2); the apical turn thus harboring more Prestin+ IHCs than the basal and middle turns. This is in accord with the higher reported Cre efficiency of Atoh1-CreER+ in the apex of the cochlea (Cox et al., 2012). We also confirmed that all Tdtomato+ (Prestin+) IHCs expressed HA (lkzf2) and vice versa (Figure 1E,F), which validated the co-expression of Ikzf2 and Tdtomato. As a point of potential interest, these results bring further support toward the ability of Ikzf2 to induce Prestin expression in IHCs. We noted that despite Tdtomato+ OHCs likely expressing both endogenous and ectopic Ikzf2, they appeared normal suggesting that these cells are able to tolerate additional Ikzf2 expression, at least until P42 (Figure 1C-C'"' and F).

Besides Prestin, overexpressing lkzf2 IHCs also upregulated a second OHC-specific marker, Oncomodulin (Ocm) (Simmons et al., 2010; Tong et al., 2016). This was only observed in the experimental mice $(n=3, P 42)$, but not in the control Rosa26 $6^{\text {CAG-LLL-kzt2/+ }}$ mice $(n=3$, P42) (Figure 1-figure supplement 3A- $\left.B^{\prime \prime}\right)$. Again, all Tdtomato+ IHCs were $\mathrm{Ocm}+$ and vice versa. Moreover, triple staining against the IHC markers, Slc7a14 (Li et alo, 2018a), Ctbp2 (a marker of synaptic ribbons) (Buran et alo, 2010; Khimich et al., 2005; Liu et al., 2014a), showed that in the control Rosa26 ${ }^{\text {CAG-LLL-kz22/+ }}$ group ( $n=3$, P42) Slc7a14 was expressed in IHCs and that the cells also contained Ctbp2+ puncta (Figure 1figure supplement $\left.3 C-C^{\prime \prime \prime}\right)$. In contrast, in the experimental mice $(n=3$, P42) Slc7a14 expression was diminished and the number of Ctbp2+ puncta was reduced in Tdtomato + IHCs (arrows in Figure 1figure supplement 3D-D"'). In Tdtomato- IHCs, Slc7a14 level and number of Ctbp2 puncta were comparable to those of the control mice as expected (dotted line in Figure 1-figure supplement 3D-D"'). Lastly, we noted that the size of the cell nuclei, which can be estimated by the area covered by Ctbp2, was smaller in Tdtomato+ IHCs than that in Tdtomato- IHCs.

Taken together, these results show that the Rosa26 $6^{\text {CAG-LSL-kzf2/+ }}$ strain was able to drive sufficient ectopic lkzf2 expression to induce endogenous IHCs to gain and lose some $\mathrm{OHC}$ and $\mathrm{IHC}$ characteristics respectively; more specifically, the $\mathrm{OHC}$ markers, Prestin and $\mathrm{Ocm}$, were found to be 


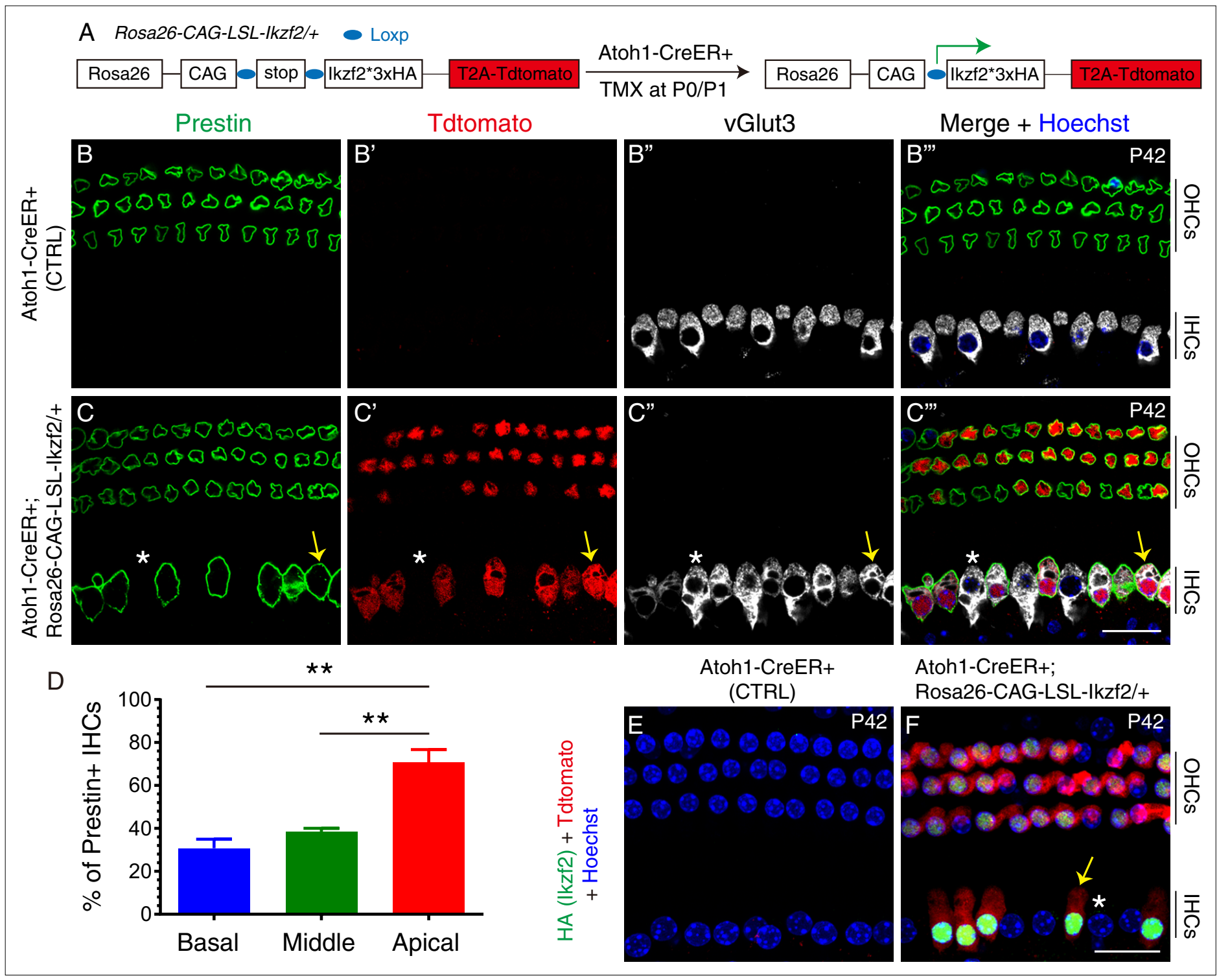

Figure 1. Prestin was expressed in IHCs upon ectopic Ikzf2 induction. (A) Illustration of approach used to induce Ikzf2 expression in HCs (both IHCs and $\mathrm{OHCs}$ ) using the Cre-LoxP system. The Atoh1-CreER+ line was used to efficiently drive Cre expression in HCs at neonatal ages when Tamoxifen (TMX) was given at P0 and P1. Tdtomato and Ikzf2 (tagged with HA) were placed in close proximity in the construct to ensure joint expression. (B-C'"') Triple labeling against Prestin, Tdtomato, and vGlut3 in P42 cochlear samples: control Atoh1-CreER+ (B-B'"') and experimental Atoh1-CreER+; Rosa26 CAG-LSL${ }^{\prime k z 22 /+}\left(\mathbf{C}-\mathbf{C}^{\prime \prime \prime}\right)$. In wild-type (B-B'"') Prestin was only expressed in OHCs; in the experimental group (C-C'"'), expression was observed in both OHCs and some IHCs. Arrows in (C-C'"'): Tdtomato+/vGlut3+/Prestin+ IHC; asterisks in (C-C'"'): vGlut3+/Tdtomato-/Prestin- IHC. (D) Quantification of Prestin+ IHCs. More Prestin+ IHCs were present in the apical turn than in basal and middle turns of the cochleae. Data was presented as mean $\pm \mathrm{SEM},{ }^{\star \star} \mathrm{p}<0.01$ (Student's t test). (E, F) Co-staining against HA (lkzf2) and Tdtomato in control (E) and experimental (F) mice at P42. HA+/Tdtomato + cells were present in experimental mice only. Arrow/asterisk in (F): IHC with/without HA (lkzf2) expression. All Tdtomato+ cells were HA+ (lkzf2 expressing) cells, and vice versa. Scale bar: $20 \mu \mathrm{m}$.

The online version of this article includes the following figure supplement(s) for figure 1:

Source data 1. It contained the numerical values of the data plotted in the graph of Figure 1D.

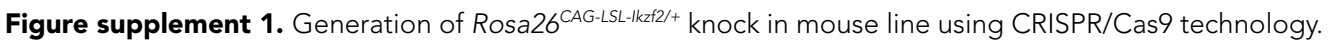

Figure supplement 2. Prestin was expressed in IHCs upon ectopic lkzf2 induction.

Figure supplement 3. IHCs with ectopic Ikzf2 expression activated $\mathrm{Ocm}$ and suppressed Slc7a14 expression, as well as exhibited fewer ribbon synapses. 
over-expressed, and IHC marker, Slc7a14, downregulated in the Tdtomato+/overexpressing Ikzf2 IHCs. In summary, we confirmed that Rosa26 $6^{\text {CAG-LSL-kkf2/+ }}$ strain could be used as a powerful genetic model for inducing functional lkzf2 expression in Cre-expressing cells.

\section{Induction of $\mathbf{l k z f 2}$ alone fails to convert adult PCs and DCs into HCs}

We next determined whether lkzf2 alone could reprogram adult cochlear SCs into Prestin+ OHCs. Fgfr3-iCreER+; Rosa26-Loxp-stop-Loxp-Tdtomato (Ai9)/+ (Fgfr3-Ai9 for short) mice were administered tamoxifen at P30/P31 and sacrificed at P60 (arrows in Figure 2A-A"). As expected, no Tdtomato+ HCs (neither IHCs nor OHCs) were observed, whilst most SCs (primarily PCs and DCs) were Tdtomato+ (inset in Figure 2A'); this was congruent with our previous reports that Fgfr3-iCreER is SC specific at adult ages (Liu et al., 2012a; Liu et al., 2012b). We also confirmed that induction of Atoh1 alone failed to convert adult cochlear SCs into HCs by analyzing Fgfr3-iCreER+; CAG-LSL-Atoh1+ mice (Fgfr3-Atoh1 for short) that were also administered tamoxifen at P30/P31 and sacrificed at P60 (Figure 2B-B" $B^{\prime \prime}$. Despite high levels of ectopic Atoh1 (tagged with HA) were observed in adult SCs (inset in Figure 2B), SCs remained Prestin- (Figure 2B"). More extensive information regarding Atoh1 expression patterns in CAG-LSL-Atoh1+ mice is available in our previous study (Liu et al., 2012a).

We next tested whether driving $\mathrm{lkzf2}$ alone in $\mathrm{SC}$ s was sufficient to induce trans-differentiation into

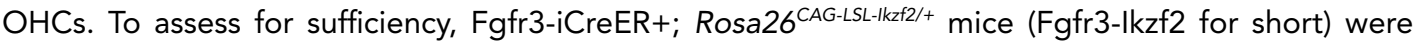
administered tamoxifen at P30/P31 and analyzed at P60. Both the $\mathrm{HC}$ layer (Figure 2C-C") and the SC layer (Figure 2D-D"') were imaged. As expected, numerous Tdtomato+ cells were observed in cochleae. As before, all Tdtomato+ cells expressed HA and vice versa (arrows in Figure 2C-D"'). However, none of these Tdtomato+/HA+ cells expressed Prestin (arrows in Figure 2C-D"'). Furthermore, we could not detect any Tdtomato+/Myo6 (HC marker)+ cells. We also noted the loss of endogenous $\mathrm{OHCs}$ (Prestin+/Tdtomato-) throughout the cochlear duct, particularly at the basal turn, which was likely a secondary effect of ectopic lkzf2 expression in adult cochlear SCs. Collectively, these results suggested that Ikzf2 alone was insufficient for converting adult cochlear SCs into nascent Myo6+ HCs or Prestin+ OHCs. Thus, in terms of cell-fate conversion, more barriers from adult cochlear $\mathrm{SCs}$ into OHCs needed to be overcome than from IHCs to OHCs.

\section{Concurrent induction of Ikzf2 and Atoh1 converts, at low efficiency, adult PCs, and DCs into Prestin+ OHC-like cells}

Considering the synergistic effects reported among TFs, such as between Six1, Atoh1, Pou4f3, and Gata3 and between Myc and Notch signaling (Chen et al., 2021; Costa et al., 2015; Menendez et al., 2020; Shu et al., 2019; Walters et al., 2017), we hypothesized that concurrent induction of Ikzf2 and Atoh1 might convert adult cochlear SCs into OHCs. We tested this by analyzing cochlear samples from Fgfr3-iCreER+; CAG-LSL-Atoh1+; Rosa26 CAG-LLL-kzf2/+ mice (Fgfr3-Atoh1-lkzf2 for short) that were given tamoxifen at P30/P31 and sacrificed at P60. As before, Tdtomato+ SCs were abundant within the OC, and $88.0 \% \pm 2.7 \%, 94.1 \% \pm 4.3 \%$, and $98.2 \% \pm 1.8 \%$ of $\mathrm{HA}+$ cells were Tdtomato + in the basal, middle, and apical turns, respectively $(n=3)$. The fact that most Tdtomato+ cells were HA+ further confirmed the high Cre activity in the Fgfr3-iCreER model. The small fraction of $\mathrm{HA}+/$ Tdtomato- cells represented populations in which ectopic Atoh1, but not lkzf2 was expressed ( $\mathrm{HA}$ is tagged to both Atoh1 and lkzf2 constructs) due to independent Cre-recombination events in the two loci. No Tdtomato+/HA- cells were observed as Tdtomato and HA are closely appositioned in the Rosa26 $6^{\text {CAG-LSL-kkf2/+ }}$ model.

Unlike in the case of exclusive Ikzf2 induction (Figure 2C-D"'), in Fgfr3-Atoh1-Ikzf2 mice $(n=3)$, Tdtomato+/Prestin+ cells were occasionally observable (arrows in Figure 2E-F"') at P60. These cells were defined as new OHC-like cells as they were derived from the original Tdtomato+ cochlear SCs (PCs and DCs) but were not identical to wild-type adult OHCs (see below). These OHC-like cells were distributed in both HC (Figure 2E-E"') and SC layers (Figure 2F-F"'). However, the number of new OHC-like cells was low, averaging to $4.7 \pm 4.2,0.7 \pm 0.3$, and $14.3 \pm 6.4$ in the basal, middle, and apical turns, respectively (Figure 2G). Moreover, Prestin protein expression was substantially lower in these OHC-like cells (blue arrows in Figure 2E-F"') compared to wild-type endogenous OHCs, the latter which expressed Prestin but not Tdtomato (yellow arrows in Figure 2F-F"'). As observed before, endogenous $\mathrm{OHC}$ loss occurred throughout the cochlear ducts (Figure 2E-F"'). These results supported the conclusion that concurrent induction of Atoh1 and Ikzf2 was sufficient to reprogram, 


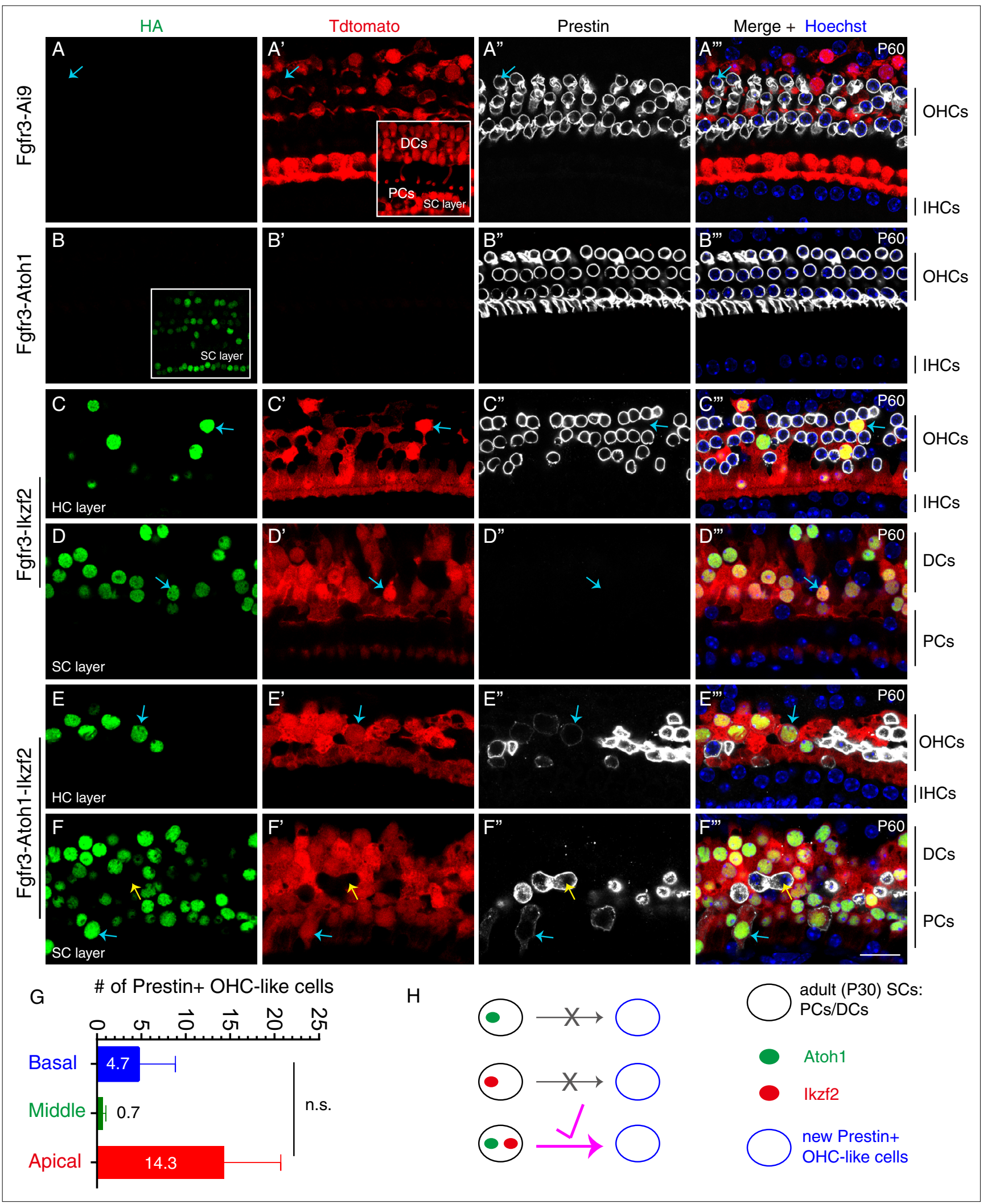

Figure 2. Joint expression of Atoh1 and Ikzf2 in adult SCs (PCs and DCs) induced transition to an OHC-like cell state at low frequency. Triple labeling against HA, Tdtomato, and Prestin in four different mouse genetic models that were administered tamoxifen at P30 and P31 and sacrificed at P60. Confocal images scanned at HC or SC layers. (A-A'") In the control Fgfr3-iCreER+; Ai9/+ (Fgfr3-Ai9) mice, Tdtomato labelled SCs (primarily PCs

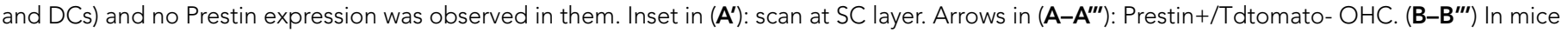
Figure 2 continued on next page 
Figure 2 continued

expressing Atoh1 in adult SCs, Fgfr3-iCreER+; CAG-LSL-Atoh1+ (Fgfr3-Atoh1), no Tdtomato signal was detected and no HA+/Prestin+ cells were observed. Inset in (B): scan at SC layer. (C-D'"') In mice expressing Ikzf2 in adult SCs, Fgfr3-iCreER+; Rosa26CAG-LSL-lkz2/+ (Fgfr3-Ikzf2), HA and Tdtomato were co-expressed in both the $\mathrm{HC}\left(\mathbf{C}-\mathbf{C}^{\prime \prime \prime}\right)$ and SC (D-D'") layer. Arrows in both layers: two cells that were HA+/Tdtomato + but did not express Prestin. Alignment of endogenous Prestin+ OHCs was abnormal and some of them were lost. (E-F"') In mice, Fgfr3-iCreER+; CAG-LSL-Atoh1+; Rosa26CAG-

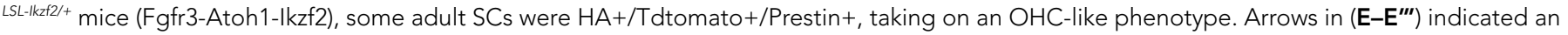
$\mathrm{OHC}$-like cell originating from an adult DC; in contrast, the blue arrows in (F-F'") indicated another OHC-like cell derived from adult PCs. Yellow arrows in (F-F"'): Prestin+/Tdtomato- endogenous $\mathrm{OHC}$ appearing in the SC layer. Prestin expression in the new OHC-like cells was noted to be lower than that in wild-type endogenous OHCs. (G) Quantification of the number of OHC-like cells throughout the cochlear turns in the Fgfr3-Atoh1-lkzf2 mice. Data was presented as mean \pm SEM $(n=3)$; no statistical difference was detected between regions (Student's $t$ test). OHC-like cells were reliably observed in all animals, but cell numbers were low and showed large variations. $(\mathbf{H})$ Summary of reprogramming outcomes in the three mice models. OHC-like cells were present only when both Atoh1 and Ikzf2 were concurrently reactivated in adult cochlear SCs. Scale bar: $20 \mu \mathrm{m}$.

The online version of this article includes the following figure supplement(s) for figure 2:

Source data 1. It contained the numerical values of the data plotted in the graph of Figure $2 \mathbf{G}$.

albeit at low efficiency, adult cochlear SCs into Prestin+ $\mathrm{OHC}$-like cells, whilst independently, neither gene was able to trigger this conversion (Figure $2 \mathrm{H}$ ). In an attempt to boost the reprogramming efficiency, we next sought to test whether pre-damaging OHCs might act as a permissive and promoting factor to SCs conversion into Prestin+ OHC -like cells.

\section{Generation of Slc26a5 $5^{\mathrm{DTR} /+}$ mouse line to study effects of OHC damage}

To non-invasively damage adult OHCs in vivo, we resorted to making use of the diphtheria toxin (DT)/ DT receptor (DTR) system, which has already been used to successfully lesion HCs in the inner ear (Cox et al., 2014; Golub et al., 2012; Tong et al., 2015). We generated a new knock-in mouse model, Slc26a5-P2A-DTR/+ (Slc26a5 $5^{D R /+}$ for short), in which the P2A-DTR fragment was inserted immediately before the stop codon (TAA) of Slc26a5 (enconding the OHC marker, Prestin) (Figure 3-figure supplement $1 \mathrm{~A}-\mathrm{C}$ ). As a result DTR expression was entirely controlled by the endogenous Slc26a5 promoter and/or enhancers and thus restricted to being expressed in OHCs, whilst still leaving $\mathrm{OHC}$ Prestin expression itself in principle unaffected. Southern blotting results confirmed the absence of random insertion of donor DNA in the host genome Figure 3-figure supplement 1D,E, and tail-DNA PCR allowed us to readily distinguish between wild-type and post-gene-targeting Slc26a5 alleles (Figure 3-figure supplement 1F).

In the absence of DT treatment, co-staining for the OHC-marker, Prestin and IHC-marker, vGlut3 revealed that OHCs were normal in P42 S/c26a $5^{\mathrm{DTR} / \mathrm{t}}$ mice $(\mathrm{n}=3)$ (Figure $3 \mathrm{~A}$ and $\left.\mathrm{A}^{\prime}\right)$. In contrast, after a single injection of DT ( $20 \mathrm{ng} / \mathrm{g}$, body weight) at P36, severe OHC loss was observed in Slc26a $5^{\mathrm{DTR} /+}$ mice $(n=3)$ at $\mathrm{P} 42$, with only a few OHCs detectable throughout the cochlear duct (arrowhead in Figure 3B'). Debris from dying OHCs were frequently observed at P42 (arrows in Figure 3B') but had disappeared by P60 (see below for details; see Figure 4). Conversely, IHCs appeared normal at P42 under DT treatment, which confirmed that DTR was indeed specifically expressed in OHCs only (Figure $3 B$ and $B^{\prime}$ ). Furthermore, the results of auditory brainstem response (ABR) measurement demonstrated that auditory thresholds at distinct frequencies in Slc26a $5^{\mathrm{DTR} /+}$ mice $(\mathrm{n}=3)$ treated with DT were significantly higher than those in untreated control Slc26a5 $5^{\mathrm{TTR} /+}$ mice $(n=3)$ (Figure 3C). In logical accord with this, the ratio of $\mathrm{OHC}$ numbers to $\mathrm{IHC}$ number was significantly reduced in Slc26a $5^{\mathrm{DTR} /+}$ mice treated with DT (red in Figure 3D) being at $\sim 0.19$, compared to the ratio of $\sim 3.23$ in control population (blue in Figure 3D). Together, these results showed that DT treatment caused, within 6 days, marked hearing impairment due to selective OHC loss, and validated the use of Slc26a $5^{\text {DTR/+ }}$ as a powerful mouse model to study the selective effects wild-type endogenous OHC loss. We also note that, thanks to the exclusive expression of Prestin in cochlear OHCs, Slc26a5 $5^{\mathrm{DTR} /+}$ mice displayed no detectable abnormalities, apart from the desired deafness following DT treatment.

\section{Endogenous OHC damage considerably enhances capacity of Ikzf2 and Atoh1 to convert adult cochlear SCs into Prestin+ OHC-like cells}

In non-mammalian vertebrates, $\mathrm{HC}$ loss triggers the regeneration of $\mathrm{HCs}$ through a cell-fate change in SCs (Janesick and Heller, 2019; Stone and Cotanche, 2007; Warchol and Corwin, 1996). This prompted us to test whether damaging endogenous wild-type adult OHCs would engender a 


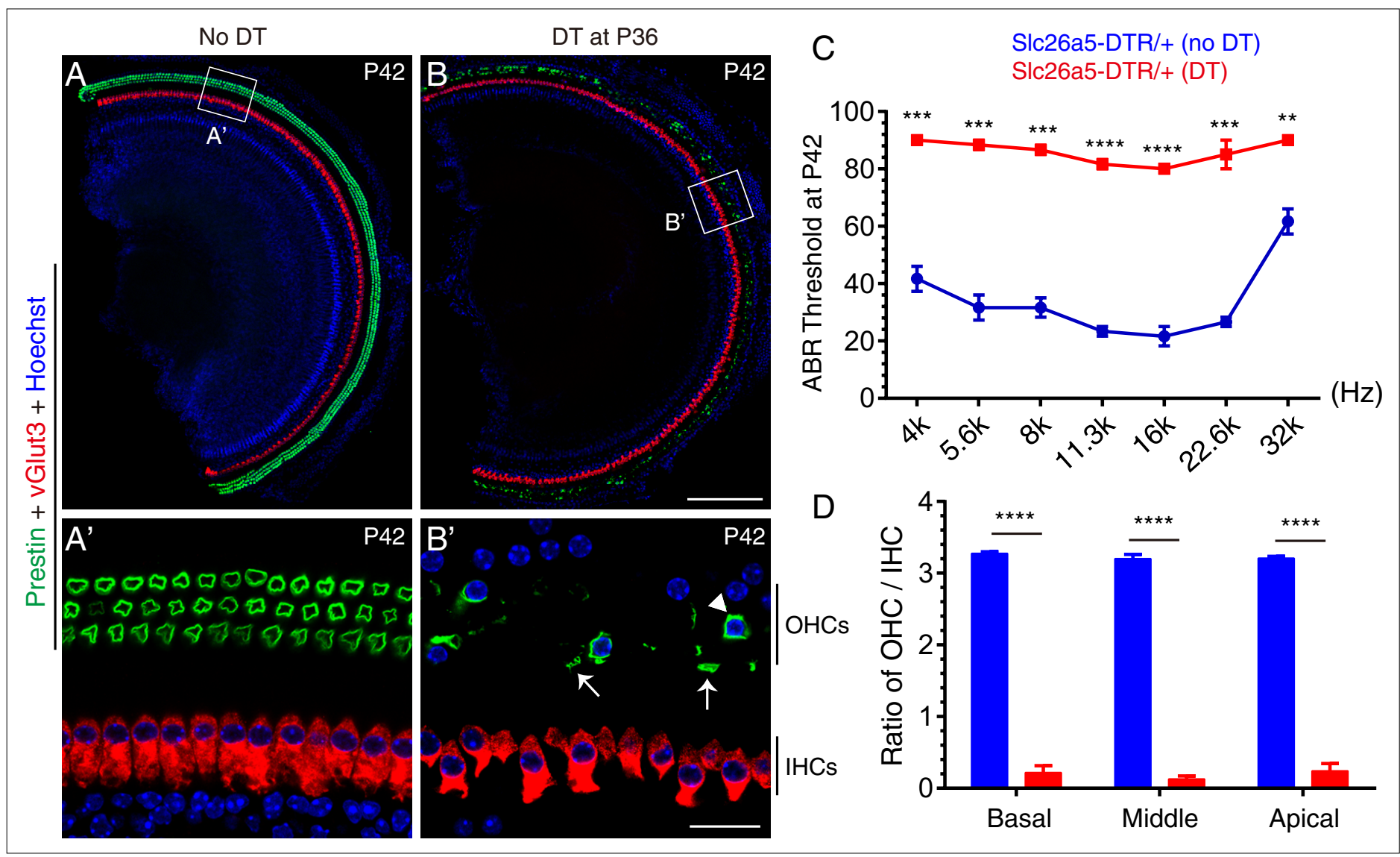

Figure 3. Targeted lesioning of adult endogenous $\mathrm{OHCs}$ via diphtheria toxin treatment. (A-B') Slc26a5 ${ }^{\text {DTR/t }}$ mice were treated without $\left(\mathbf{A}-\mathbf{A}^{\prime}\right.$, control) or

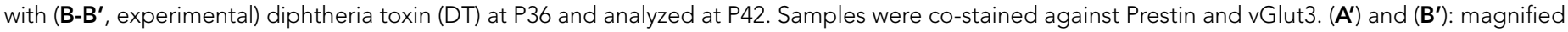
images of boxed region in (A) and (B). DT treatment led to rapid OHC death within 6 days; only a few OHCs remained (arrowhead in $B^{\prime}$ ); debris of dying $\mathrm{OHCs}$ were frequently detected (arrows in $\mathrm{B}^{\prime}$ ). Much of the green signal in (B) came from the dying OHC debris. (C) Auditory brainstem response (ABR) threshold measurement of SIc26a5 $5^{D T R /+}$ mice treated with (red line) or without (blue line) DT. After DT treatment ABR thresholds were significantly higher throughout the frequency ranges. (D) Ratios of OHCs to IHCs within a same scanning area in control mice (blue) and experimental mice (red); relative $\mathrm{OHC}$ numbers were significantly reduced in the experimental mice. Data was presented as mean \pm SEM $(n=3)$. Student's t-test were run for statistical testing. ${ }^{\star \star} p<0.01,{ }^{* \star \star} p<0.001,{ }^{* \star \star \star} P<0.0001$. Scale bars: $200 \mu \mathrm{m}$ (B), $20 \mu \mathrm{m}\left(\mathbf{B}^{\prime}\right)$.

The online version of this article includes the following figure supplement(s) for figure 3 :

Source data 1. It contained the numerical values of the $A B R$ thresholds presented in Figure $3 C$

Source data 2. It contained the numerical value of the ratio of OHCs to IHCs described in Figure 3D.

Figure supplement 1. Generation of the Slc26a5 $5^{\text {TR/+ }}$ knockin mouse model.

more favorable environment for SC transdifferentiation, thereby potentially increasing the conversion efficiency of adult cochlear SCs overexpressing lkzf2 and Atoh1 into OHC-like cells. For this, we compared four genetic mouse strains: (1) Slc26a5 ${ }^{\mathrm{DTR} /+}$; (2) Fgfr3-iCreER+; CAG-LSL-Atoh1+; Slc26a5 $5^{\text {DTR/+ }}$ (Fgfr3-Atoh1-DTR); (3) Fgfr3-iCreER+; Rosa26 ${ }^{\text {CAG-LLL-kzf2/+ }}$ Slc26a5 $5^{\text {DTR/+ }}$ (Fgfr3-lkzf2-DTR);

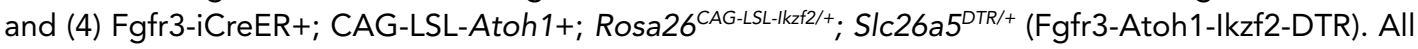
mice were first injected tamoxifen at P30 and P31, to trigger expression of Atoh1 or Ikzf2 or both or non. Six days later, at P36, DT was administered to trigger OHC damage (Figure 4A,B). By injecting Tamoxifen, prior to DT, we could selectively and permanently label adult SCs (mainly PCs and DCs) with $\mathrm{HA}$ and Tdtomato before any $\mathrm{OHC}$ damage. The reverse order was not used so as to avoid the possibility of $\mathrm{OHC}$ damage leading to changes in the Cre-expression pattern in Fgfr3-iCreER+ mice.

Akin to before, P60 Slc26a5 $5^{\text {DTR/+ }}$ mice treated with DT had few visible OHCs (arrows in Figure 4D) as compared to the untreated, same aged, Slc26a5 $5^{D T R /+}$ control mice (Figure 4C). However, unlike the findings at $\mathrm{P} 42$, at $\mathrm{P} 60$ little $\mathrm{OHC}$ debris was observed. Furthermore, $\mathrm{HA}+/$ Prestin + cells were identified in neither Fgfr3-Atoh1-DTR mice (Figure 4E) nor Fgfr3-Ikzf2-DTR mice (Figure 4F) at P60, 


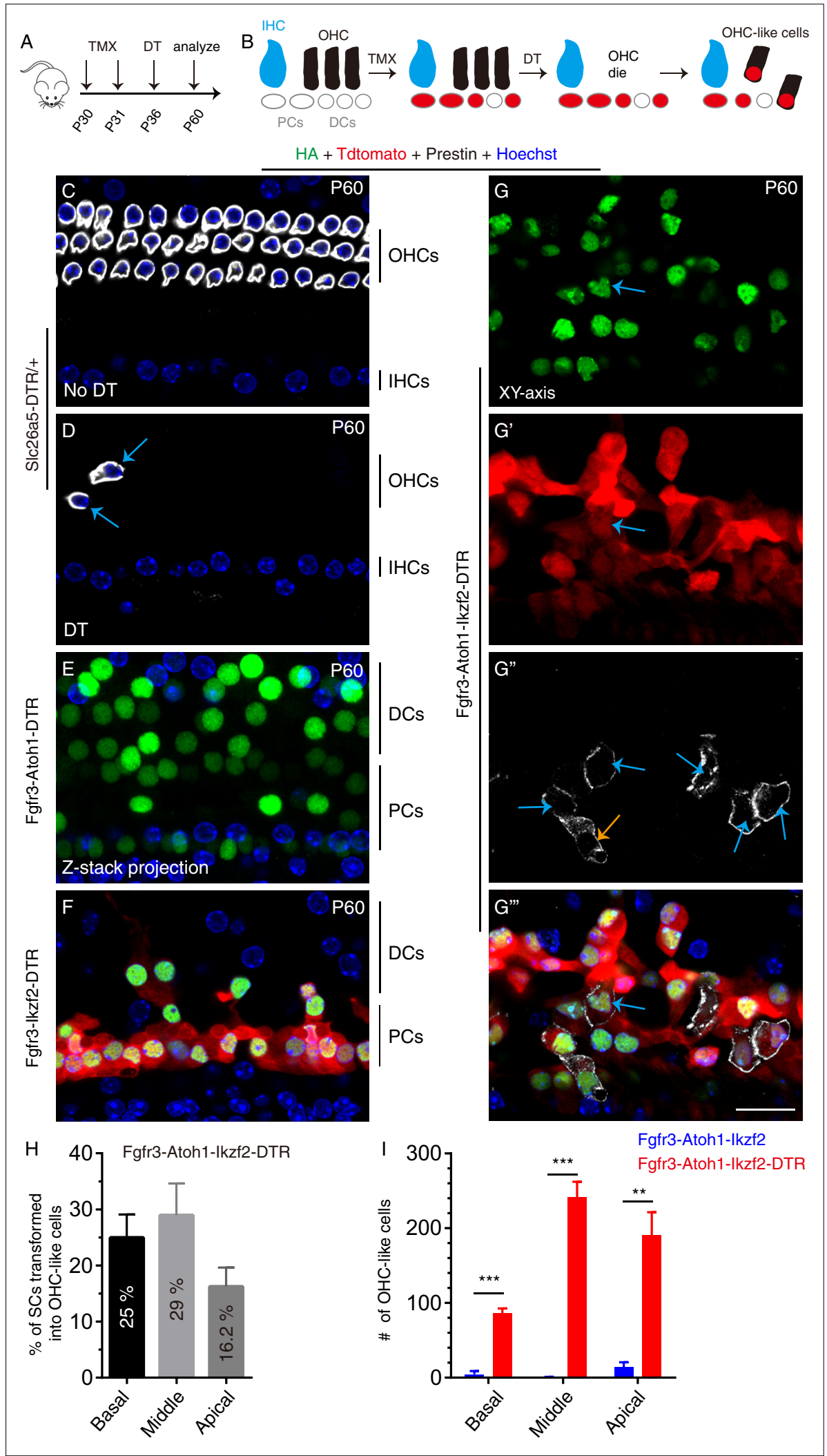

Figure 4. Lesion to endogenous OHCs enhances reprogramming efficiency of adult SCs by Atoh1 and Ikzf2. (A) Three distinct mice models were subject to tamoxifen (TMX) treatment at P30 and P31, followed by DT treatment at P36 and analysis at P60. (B) Illustration of key cellular events occurring during the experiment: induction of Atoh1 and Ikzf2 in adult PCs and DCs, which were permanently labeled with Tdtomato for the subsequent fate-mapping

Figure 4 continued on next page 
Figure 4 continued

analysis. (C-G'"') Triple labeling against HA, Tdtomato, and Prestin in four models: (1) Slc26a5 $5^{\text {TR/+ }}$ (C, D), (2) Fgfr3iCreER+; CAG-LSL-Atoh1+; Slc26a5 $5^{\text {DTR/+ }}$ (Fgfr3-Atoh1-DTR; E), (3) Fgfr3-iCreER+; Rosa26 $6^{\text {CAG-LLL-kzt2/+; Slc26a5 }} 5^{\text {DTR/+ }}$ (Fgfr3-Ikzf2-DTR; F), and (4) Fgfr3-iCreER+; CAG-LSL-Atoh1+; Rosa26 CAG-LLL-lkf2/+; Slc26a5 ${ }^{\text {DTR/+ }}$ (Fgfr3-Atoh1-Ikzf2DTR; G-G'"). Relative to Slc26a5 $5^{\text {TR/+ }}$ mice not treated with DT (C), DT-treated Slc26a5 ${ }^{\text {DTR/+ }}$ mice harbored fewer normal Prestin + OHCs at P60 (arrows in D). Debris of dying OHCs had disappeared. No OHC-like cells were observed in the first three models, but Tdtomato $/ / \mathrm{HA}+/$ Prestin $+\mathrm{OHC}$-like cells (orange and blue arrows in G") were present in the Fgfr3-Atoh1-Ikzf2-DTR model (G-G'"). (H) Percentages of SCs that were transformed into OHC-like cells at different cochlear turns in Fgfr3-Atoh1-Ikzf2-DTR mice. (I) Comparison of OHC-like cell numbers between Fgfr3-Atoh1-Ikzf2-DTR and Fgfr3-Atoh1-Ikzf2 models (without damaging adult wild-type OHCs). Fgfr3-Atoh1-Ikzf2-DTR mice harbored considerably more OHC-like cells than Fgfr3-Atoh1-Ikzf2 mice. Data was presented as mean \pm SEM $(n=3)$. Student's t test was used for statistical analysis. ${ }^{\star \star} p<0.01,{ }^{\star \star \star} p<0.001$. Scale bar: $20 \mu \mathrm{m}$.

The online version of this article includes the following figure supplement(s) for figure 4:

Source data 1. It contained the numerical values of the data plotted in the graph of Figure $4 \mathbf{H}$.

Source data 2. It contained the numerical values of the data plotted in Figure 4I.

Figure supplement 1. Visualization of $\mathrm{OHC}$-like cells in orthogonal projections.

Figure supplement 2. Nascent HCs emerged at P42 and OHC-like cells at P46.

Figure supplement 2-source data 1. It contained the number of new HCs at P42 and P46 presented in the graph of Figure 4-figure supplement 2D.

Figure supplement 2-source data 2. It contained the numbers of new HCs at P46 presented in the graph of Figure 4-figure supplement 2E.

suggesting that Atoh1 or Ikzf2 alone was insufficient to convert mature SCs into OHC-like cells after endogenous $\mathrm{OHC}$ damage.

In contrast, in P60 Fgfr3-Atoh1-lkzf2-DTR mice, Tdtomato+/HA+/Prestin+ OHC -like cells were frequently observed. Confocal scanning of the entire cochlear duct $(n=3)$ revealed the presence of $359.3 \pm 46.2,878.0 \pm 118.7$, and $1195 \pm 81.6$ Tdtomato $+/ H A+$ cells of which $86.3 \pm 6.3,241.0 \pm 21.1$, and $190.3 \pm 31.1$ cells were new Prestin+ OHC -like cells (arrows in Figure 4G-G"', Figure 4-figure supplement 1A-B"' and Video 1) in the basal, middle, and apical turns, respectively. Or in other words, $25.0 \% \pm 4.1 \%, 29.0 \% \pm 5.7 \%$, and $16.2 \% \pm 3.4 \%$ of adult cochlear tdTomato + SCs transdifferentiated into $\mathrm{OHC}$-like cells in the basal, middle, and apical turns, respectively (Figure 4H). This rate was significantly greater (Figure 4I) than that obtained with un-lesioned P60 Fgfr3-Atoh1-Ikzf2 mice (Figure 2E-G). Expression levels of Prestin were also higher in mice with pre-lesioned $\mathrm{OHCs}$ (Figure 4G-G"), but still remained considerably lower than those in wild-type endogenous OHCs (Figure $4 \mathrm{C}$ vs Figure $4 \mathrm{G}^{\prime \prime}$ ). In addition, $~ 82.6 \%$ of OHC-like cells were located in the top HC layer (blue arrows in Figure 4G", Figure 4-figure supple-

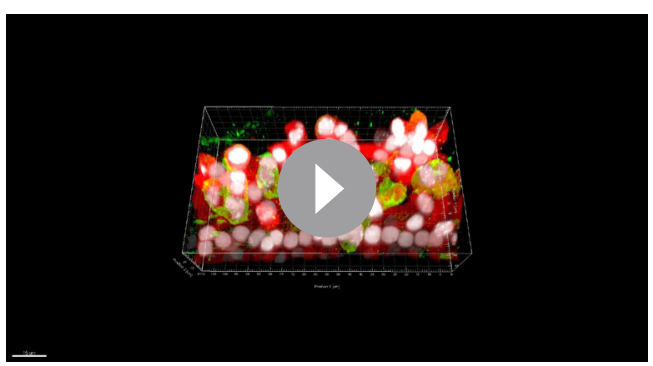

Video 1. The confocal data presented in Figure 4GG'" and Figure 4-figure supplement 1A-A'"' was converted to Imaris format and loaded in the Imaris software to more readily visualize $\mathrm{OHC}$-like cells using orthogonal projections of the data. Red, green, and white colors represented Tdtomato signal and Prestin and $\mathrm{HA}$ labelling respectively. OHC-like cells were defined as triple-positive cells.

https://elifesciences.org/articles/66547/figures\#video1 ment $\left.1 \mathrm{~A}-\mathrm{A}^{\prime \prime \prime}\right)$, whereas the rest remained in the bottom SC layer (orange arrows in Figure 4G", Figure 4-figure supplement 1B-B'"'). These results demonstrated that damaging endogenous OHCs markedly enhanced the reprogramming ability of Ikzf2 and Atoh1 to convert adult cochlear SCs into OHC-like cells.

\section{The conversion of SCs to OHC- like cells involves an intermediate general HC state}

We next determined the time it took for adult cochlear SCs to form nascent $\mathrm{HCs}$ and $\mathrm{OHC}$-like cells (Figure 4-figure supplement 2A-C"'). Nascent HCs were defined as Tdtomato+/Myo6+/ Prestin- (arrowheads in Figure 4-figure supplement 2B-B"'). OHC-like cells were defined as 
Tdtomato+/Myo6+/Prestin+. P42 was the earliest age at which the nascent HCs were detected; Myo6 expression was weak at this stage. Scanning the entire cochlear duct at $\mathrm{P} 42$ revealed the presence of only $29.0 \pm 13.5,66.0 \pm 35.23$, and $52.7 \pm 25.4$ nascent $\mathrm{HCs}$ throughout the basal, middle, and apical turns $(n=3)$, respectively (black in Figure 4-figure supplement 2D). No Tdtomato+/Myo6+/ Prestin+ OHC- like cells were detected at P42.

Four days later, at P46 $(n=4)$, there were $56.5 \pm 28.8,118.0 \pm 61.2$, and $111.0 \pm 57.3$ new HCs that were Tdtomato+/Myo6+ (gray in Figure 4-figure supplement 2D,F), and, of these, $27.8 \pm$ $15.0,43.5 \pm 25.3$, and $36.8 \pm 21.0$ were Tdtomato+/Myo6+/Prestin+ (classified as OHC-like cells; green in Figure 4-figure supplement 2E); thus, OHC-like cells accounted for $49.2 \%$ (27.8/56.5), $36.9 \%(43.5 / 118)$, and $33.2 \%$ (36.8/111) of total new HCs in basal, middle, and apical turns, respectively. As mentioned, the remaining Tdtomato+/Myo6+/Prestin- cells were defined as nascent HCs, and the Tdtomato+/Myo6-/Prestin- cells were defined as 'SCs failing to become HCs'. Notably, we sorted new HCs into nascent $\mathrm{HCs}$ and $\mathrm{OHC}$-like cells based solely on absence or presence of Prestin. Among the four mice analyzed, two mice harbored substantially fewer Tdtomato+/Myo6+ cells than the others, which caused the large variations in numbers; nevertheless, the overall trend was that the larger the number of Tdtomato+/Myo6+ cells, the larger the number of Tdtomato+/Myo6+/Prestin+ $\mathrm{OHC}$-like cells. Importantly, no Tdtomato+/Prestin+ cells lacking Myo6 expression were detected. Together, these findings suggested that the generation of new OHC-like cells generally involved an initial cell-fate transition from SCs (PCs and DCs) into nascent HCs, which occurred by P42 (12 days
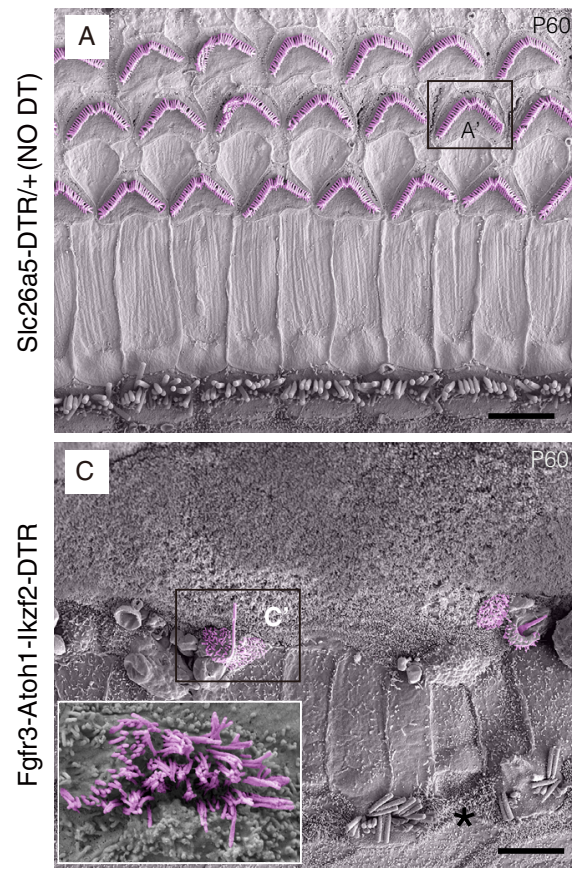
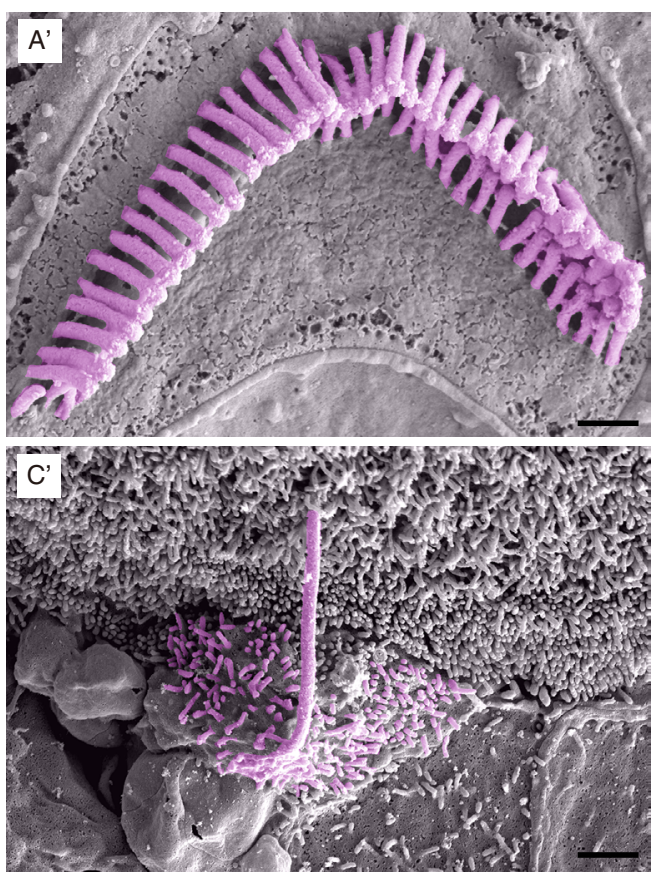

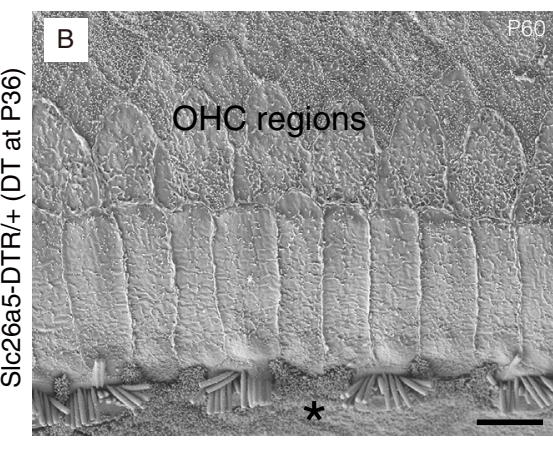

SIc26a5-DTR/+ (no DT) SIc26a5-DTR/+ (DT) Fgfr3-Atoh1-Ikzf2-DTR

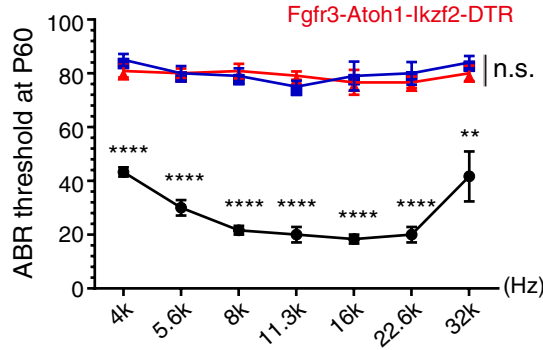

Figure 5. Hair bundles were present in OHC-like cells. Scanning electron microscopy (SEM) analysis of samples from three different mouse models at P60. (A-A') OHCs harbored V- or W-shaped hair bundles in S/c26a5 $5^{\text {DTR/+ }}$ mice not treated with DT. (A'): high-magnification view of boxed region in (A). (B) Majority of OHCs had disappeared by P60 in Slc26a5 $5^{D T R /+}$ mice upon DT treatment at P36. Black asterisk: one IHC that was absent. (C) Immature

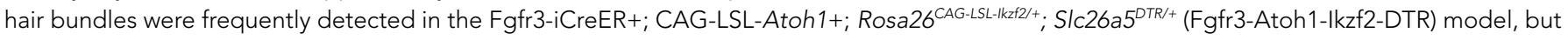

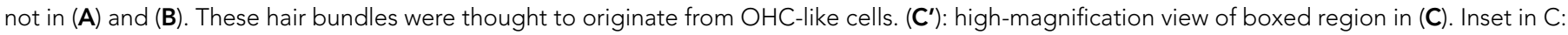
instance of occasional hair bundles. Black asterisk: one IHC that was missing. (D) ABR measurements of the three mice models. Relative to the untreated Slc26a5 ${ }^{\text {TTR/t }}$ control mice (black line, $n=3$ ), the ABR thresholds of Slc26a5 $5^{\text {TR/ }}$ treated with DT (blue line, $\left.n=5\right)$ and in Fgfr3-Atoh1-Ikzf2-DTR mice (red line, $n=6$ ) were significantly higher. The blue and red lines showed no statistically significant difference at any frequency (n.s.). Data was presented as mean \pm SEM. Student's t-test was used for statistical testing. ${ }^{* \star} \mathrm{p}<0.01,{ }^{\star \star \star \star} \mathrm{p}<0.0001$. Scale bars: $5 \mu \mathrm{m}(\mathbf{A}-\mathbf{C}), 1 \mu \mathrm{m}\left(\mathbf{C}^{\prime}\right)$, and $500 \mathrm{~nm}\left(\mathbf{A}^{\prime}\right)$.

The online version of this article includes the following figure supplement(s) for figure 5 :

Source data 1. It contained the numerical values of the ABR thresholds described in Figure 5D. 
after Atoh1 and Ikzf2 gene induction, and 6 days after DT treatment), and that this was followed by a second transition from nascent $\mathrm{HCs}$ into $\mathrm{OHC}$-like cells.

\section{New OHC-like cells are able to produce hair bundles}

Scanning electron microscopy (SEM) was used to ascertain the presence and morphology of hair bundles (a cluster of stereocilia) of the Prestin+ new OHC-like cells at P60 (Figure 5). These characteristic staircase-shaped hair bundles are where mechanoelectrical transduction (MET) channels critical for hearing reside (Corey and Holt, 2016; Douguet and Honore, 2019; Wu and Muller, 2016). In non-DT-treated control Slc26a5 $5^{\text {DTR/+ }}$ mice, the stereotyped V- or W-shaped hair bundles were present in $\mathrm{OHCs}$ (Figure 5A,A'), whereas very few hair bundles remained in Slc26a5 $5^{\mathrm{DTR} /+}$ mice treated with DT

(Figure 5B). This was consistent with the results from the immunostaining assays (Figure 4D).

Intriguingly, in Fgfr3-Atoh1-Ikzf2-DTR P60 mice, we frequently detected stereocilia with a single long bundle, lacking the staircase shape (Figure $5 C-C^{\prime}$ ). Stereocilia with several long bundles were seldom observed (inset in Figure 5C). These rare hair bundle likely belonged to OHC-like cells (or nascent HCs), as such hair bundles were never observed in Slc26a5 $5^{\mathrm{DTR} /+}$ mice, irrespective of DT treatment (Figure 5A,B). Auditory brain response (ABR) measurement showed that the thresholds at distinct frequencies were markedly higher in Fgfr3-Atoh1-lkzf2-DTR mice ( $n=6$, red line in Figure 5D) compared to non-DT-treated Slc26a5 $5^{D T R /+}$ mice $(n=3$, black line in Figure 5D). Regretfully, no hearing improvement (lowering of threshold) was apparent between Fgfr3-Atoh1-lkzf2-DTR compared to DT-treated Slc26a5 $5^{\mathrm{DTR} /+}$ mice ( $n=5$, blue line in Figure 5D). Notably, we observed IHC loss at P60 (asterisks in Figure 5B,C). This could be a secondary effect of $\mathrm{OHC}$ damage, since no IHC death was observed at P42 (Figure 3). Collectively, these results showed that the new OHC-like cells still differed from adult endogenous OHCs in terms of hair-bundle abundance and morphology as well as Prestin expression levels, and were not yet functional. The degree to which $\mathrm{OHC}$-like cells resembled adult endogenous $\mathrm{OHCs}$ was investigated next through single-cell RNA-Seq analyses.

\section{Single-cell RNA-Seq reveals genes selectively enriched in adult wild- type OHCs and SCs}

To perform single-cell RNA-Seq on adult OHCs and SCs, we manually picked 17 wild-type Tdtomato+ $\mathrm{OHCs}$ from Slc26a5 $5_{\mathrm{CreER} /+}$; Ai9/+ mice at P30 and 16 wild-type Tdtomato+ SCs (primarily PCs and DCs) from Fgfr3-iCreER+; Ai9/+ mice at P60 (Figure 6A). Slc26a5 ${ }^{\mathrm{CreER/+}}$ is a knock-in mouse strain with $\mathrm{OHC}$-specific Cre activity (Fang et al., 2012). All cells were identified based on their endogenous Tdtomato fluorescence and were picked and washed thrice under a fluorescence microscope before final placement in PCR tubes. RNA-Seq libraries were then prepared using the Smart-Seq approach (Figure 6A). Manual picking combined with Smart-Seq has been successfully used in previous geneprofiling studies on adult cochlear HCs and SGNs (Li et al., 2020b; Liu et al., 2014b; Shrestha et al., 2018).

We first compared gene profiles between adult endogenous OHCs and SCs, and identified 1051 and 1982 genes enriched respectively $(p<0.001)$ in adult OHCs and SCs (Figure 6-figure supplement 1A); Supplementary file 1 contained the full list of enriched genes. The list of these adult $\mathrm{OHC}$ enriched genes (OHC genes for short) included previously known highly expressed adult OHC genes, such as Myo7a, Ocm, Slc26a5 (Prestin), Ikzf2, Espn, Tmc1, Cib2, Lhfpl5, Lmo7, Lbh, and Sri (Chessum et al., 2018; Du et al., 2019; Giese et al., 2017; Liu et al., 2014b; Ranum et al., 2019; Xiong et al., 2012; Zheng et al., 2000). Similarly, previously known pan-SC markers, such as Sox2 and Sox10, and two recently identified DC-specific genes, Bace2 and Ceacam16 (Li et al., 2018b; Ranum et al., 2019), were found amongst the adult SC-enriched genes (SC genes for short). Gene Ontology (GO) analysis showed that $\mathrm{OHC}$ genes were involved in sensory perception of sound, inner ear morphogenesis and stereocilium organization (Figure 6-figure supplement 1B). OHC genes associated with each GO category were summarized in Supplementary file 2. These results showed that the picked adult endogenous $\mathrm{OHCs}$ and SCs were pure and that our single cell RNA-Seq data was of high quality. These differentially enriched genes identified in $\mathrm{OHCs}$ and $\mathrm{SC}$ s were subsequently used as reference to assess the degree of cell fate conversion of SC-derived nascent $\mathrm{HCs}$ and $\mathrm{OHC}$-like cells.

Some unexpected findings from the RNA-Seq data included Myo6 expression levels: although Myo6 protein is known to be enriched in OHCs, Myo6 mRNA was not significantly enriched in adult OHCs, likely because Myo6 mRNA was also detected (albeit at a lower level) in adult SCs. Myo6 


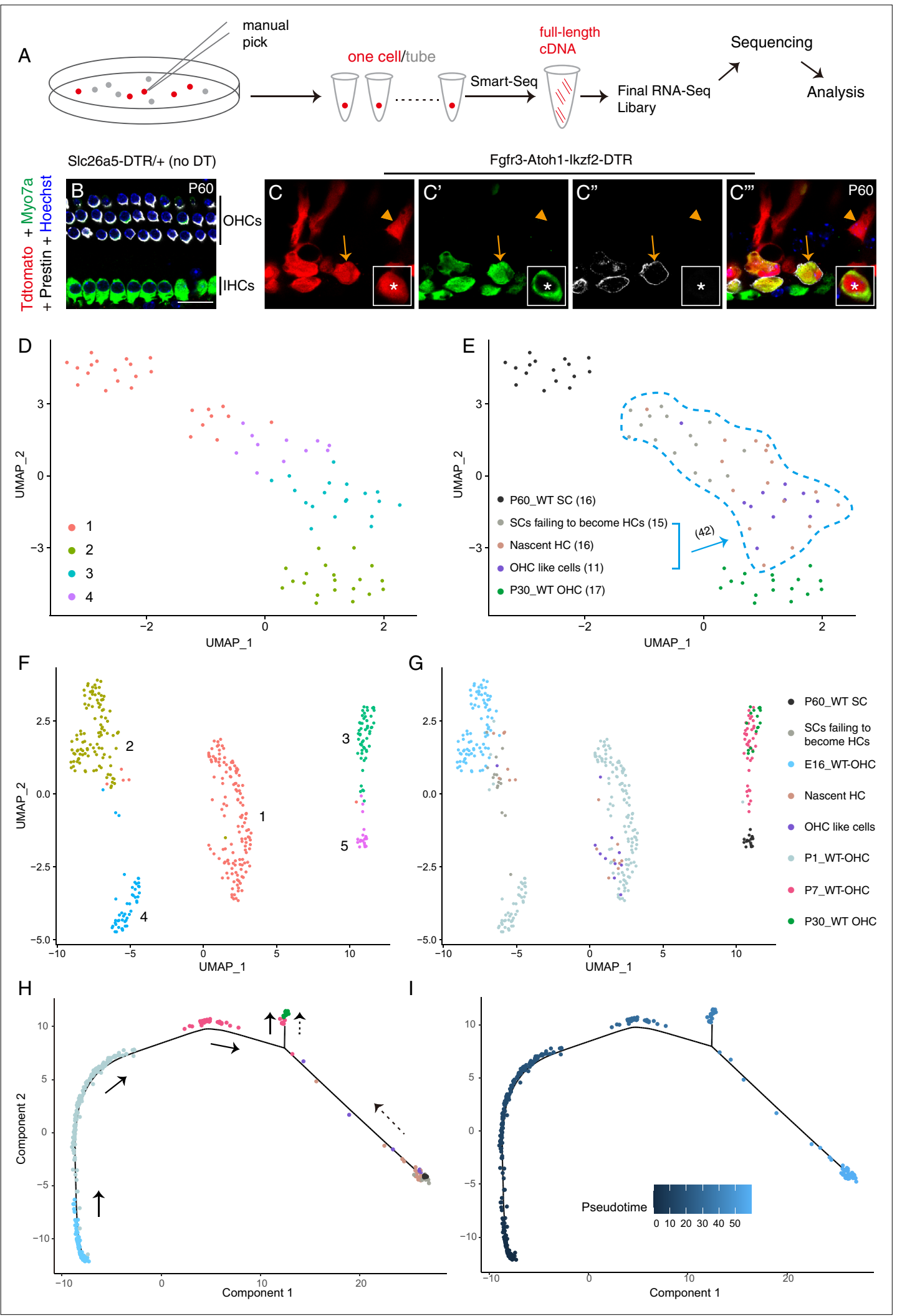

Figure 6. OHC-like cells were most akin to P1 wild-type OHCs. (A) Illustration of the single cell RNA-Seq experimental pipeline. (B-C'"') Triple labeling against Myo7a (maker for HCs), Prestin, and Tdtomato in cochlear samples from control (B) and Fgfr3-Atoh1-Ikzf2-DTR (C) mice at P60. Arrows: Tdtomato+/Myo7a+/Prestin+ OHC -like cells; arrowheads: Tdtomato+/Myo7a-/Prestin- cell, defined as SCs failing to become HCs; asterisks: nascent HC that was Tdtomato+/Myo7a+/Prestin- (inset in C-C'"). (D-E) UMAP embedding of RNA-seq data revealed four main clusters (D). The same plot Figure 6 continued on next page 
Figure 6 continued

was presented with the five different cell types included in the data labelled separately (E). The 42 Tdtomato+ cells within the light-blue dotted lines (E) originated from the P60 Fgfr3-Atoh1-lkzf2-DTR mice. When unsupervised clustering was performed over all 42 Tdtomato+ cells, three clusters were identified. (F-G) UMAP analysis of cells in (D-E) as well as wild-type OHCs at E16, P1, and P7 (E16_WT OHCs, P1_WT OHCs, and P7_WT OHCs; further information was presented in Figure 6-figure supplement 5). OHC-like cells were overlapped with P1_WT OHCs. (H-I) Trajectory analysis of all cells in (F-G). Black arrows in (H) represented the developmental trajectory of endogenous OHCs between E16 and P30, whilst the black dotted arrows marked the reprogramming trajectory from endogenous adult SCs to endogenous adult OHCs. Scale bar: $20 \mu \mathrm{m}$.

The online version of this article includes the following figure supplement(s) for figure 6:

Figure supplement 1. Transcriptomic comparison between adult wild-type OHCs and SCs.

Figure supplement 2. OHC-like cells upregulated $729 \mathrm{OHC}$ genes and downregulated $331 \mathrm{SC}$ genes.

Figure supplement 3. Two trajectory analyses by different cell pooling.

Figure supplement 4. Heterogeneous expression of $\mathrm{OHC}$ genes across $\mathrm{OHC}$-like cells.

Figure supplement 5. Trajectory analysis of wild type differentiating OHCs.

Figure supplement 6. $\mathrm{OHC}$-like cells primarily expressed neonatal $\mathrm{OHC}$, but minimally expressed neonatal $\mathrm{IHC}$ or utricle $\mathrm{HC}$ genes.

Figure supplement 7. Transcriptomic differences between OHC-like cells and mature OHCs.

mRNA is also known to be present in other non-HC populations (Kolla et al., 2020; Scheffer et al., 2015). Also noteworthy, Myo7a was significantly enriched in adult OHCs (red arrow in Figure 6figure supplement 1A) and also highly expressed in nascent $\mathrm{HCs}$ and $\mathrm{OHC}$-like cells; however, this was not the case in SCs failing to become HCs (Figure 6B-C"'). This led us to use Myo7a as an early $\mathrm{HC}$ marker and as a means of defining general HC fate in the RNA-Seq analysis described below.

\section{OHC-like cells upregulate OHC genes and downregulate SC genes}

We next focused on comparing global gene profiles between $\mathrm{OHC}$-like cells, adult endogenous OHCs, and SCs. We manually picked 42 Tdtomato+ cells from Fgfr3-Atoh1-lkzf2-DTR mice at P60 (Figure 6A) and sorted them into three types: (1) Tdtomato+/Myo7a+/Prestin+ cells (defined as OHC-like cells, arrows in Figure 6C-C"In; $n=11$ cells); (2) Tdtomato+/Myo7a+/Prestin- cells (defined as nascent HCs, asterisks in inset of Figure 6C-C'" $n=16$ ); and (3) Tdtomato+/Myo7a-/Prestincells (defined as SCs failing to become HCs, arrowheads in Figure $6 C^{-} C^{\prime \prime \prime} ; n=15$ ). The proportion of OHC-like cells was $26.2 \%(11 / 42)$, which agreed with the immunostaining results $(16.2 \%-29.0 \%)$ (Figure 4H), giving us confidence in the suitability of this criterion. Moreover, expression of Atoh1 and Ikzf2 (arrows in Figure 6-figure supplement 2A) was enriched in all 42 cells, but not in adult endogenous SCs. This also confirmed that Atoh1 and Ikzf2 were permanently overexpressed in the 42 Tdtomato+ cells, regardless of cell fate.

UMAP (uniform manifold approximation and projection) analysis demonstrated that OHC-like cells differed more greatly from adult endogenous P60 SCs (hereafter named as WT_P60 SCs) and were more similar to P30 adult endogenous OHCs (hereafter named as WT_P30 OHCs) than SCs failing to become HCs (Figure 6D,E). Besides Slc26a5 (Prestin) and Myo7a (purple arrows in Figure 6-figure supplement 2A), 1313 genes were expressed at a significantly higher level in $\mathrm{OHC}$-like cells relative to WT_P60 SCs. GO analysis of these 1313 genes revealed that they were associated with functions involving sensory perception of sound (Figure 6-figure supplement 2B), which further supported the notion that $\mathrm{OHC}$-like cells globally behave like HCs. Supplementary file 3 included all genes associated with the GO analysis. Notably, $55.5 \%$ (729/1313) of the genes expressed more in OHC-like cells than WT_P60 SCs overlapped with OHC genes, such as Myo7a, Pvalb, Calb1, Cib2, and Lhfpl5 (purple arrows in Figure 6-figure supplement 2A). Conversely, 527 genes were expressed at significantly lower levels in OHC-like cells as compared to WT_P60 SCs, of which 62.8 \% (331/527) overlapped with SC genes, such as Tuba1b, Gjb2, and Rorb (black arrows in Figure 6-figure supplement 2A). Supplementary file 4 contained the entire list of genes that were differently expressed between OHC-like cells and WT_P60 SCs, as well as the 729 OHC genes that were upregulated, and 331 SC genes that were down-regulated in $\mathrm{OHC}$-like cells. Furthermore, we also included data from nascent $\mathrm{HCs}$ and SCs failing to become HCs for completeness and as potential intermediate cell types. In general, the identified genes above were either not or only slightly upregulated or downregulated in SCs failing to become HCs (Figure 6-figure supplement 2A). Together, this suggested that $\mathrm{OHC}$ like cells were on an $\mathrm{OHC}$-lineage differentiation track. 
To our surprise, trajectory analysis including the $42 \mathrm{Tdtomato}+$ cells suggested that $\mathrm{OHC}$-like cells and nascent HCs were closer to each other, relative to SCs failing to become HCs (Figure 6figure supplement $3 \mathrm{~A}, \mathrm{~B}$ ). Furthermore, another trajectory analysis confirmed that $\mathrm{OHC}$-like cells and nascent HCs were closer to WT_P30 OHCs, and conversely SCs failing to become HCs were closer to WT_P60 SCs (Figure 6-figure supplement $3 C_{1} D$ ). The transcriptomic similarity between OHC-like cells and nascent HCs supported that, despite Prestin expression only being present in $\mathrm{OHC}$-like cells, the general expression patterns of other $\mathrm{HC}$ genes might be quite similar. This possibility was partially supported by the finding that not all Prestin+ $\mathrm{OHC}$-like cells expressed the early pan-HC markers, Rbm24, Pvalb, and Calb1, according to both RNA-Seq and immunostaining results (Figure 6-figure supplements 2 and 4). These pan-HC markers are typically expressed earlier than Prestin in endogenous OHCs (Grifone et al., 2018; Li et al., 2018b; Liu et al., 2012a; Wang et al., 2021). Together, our results showed that OHC-like cells/nascent HCs resembled WT_P30 OHCs considerably more than SCs failing to become HCs. Next, we evaluated the differentiation status of the OHC-like cells.

\section{OHC-like cells resemble wild-type neonatal differentiating OHCs}

By reanalyzing previously published data from a recent single-cell RNA-Seq studies covering wild-type cochlear OHCs, IHCs, SCs, GER (greater epithelial ridge) cells, and LER (lesser epithelial ridge) cells (Kolla et al., 2020), we could compare OHC-like cell differentiation status at finer granularity. More specifically, we extracted from these data OHCs at three different ages: E16 $(n=87)$, P1 $(n=170)$, and P7 ( $n=39$ ) (Figure 6-figure supplement 5A). These pooled OHCs were highly heterogeneous, especially at P1, due in part to the basal-to-apical and medial-to-lateral developmental gradient in the cochlear duct, as revealed by trajectory analysis of these OHCs (Figure 6-figure supplement 5B, B'). These E16, P1, and P7 OHCs were pooled with our own OHC-like cells, nascent HCs, SCs failing to become HCs, WT_P30 OHCs, and WT_P60 SCs to form a new dataset. Five main clusters were identified (Figure 6F): cluster 1 included 9/11 OHC-like cells, 11/16 nascent HCs, and the majority of wildtype OHCs at P1 (WT_P1 OHCs) (Figure 6G). Cluster 2 consisted of 2/11 OHC-like cells, 5/16 nascent HCs, 12/15 SCs failing to become HCs, and wild-type OHCs at E16 (WT_E16 OHCs). Majority of wildtype OHCs at P7 (WT_P7 OHCs) and WT_P30 OHCs formed cluster 3, suggesting that P7 OHCs were already well differentiated (Jeng et al., 2020). Cluster 4 contained the remaining fraction of WT_P1 $\mathrm{OHCs}$ and 3/15 SCs failing to become HCs, while majority of cells in cluster 5 were WT_P60 SCs. UMAP analysis suggested that OHC-like cells generally resembled WT_P1 OHCs the most. Further support for this came from the presence of Insm1 mRNA and protein in OHC-like cells (Figure 6figure supplement 2A, and Figure 6-figure supplement 4A-B'"'); Insm1 is a gene that is only transiently expressed in differentiating $\mathrm{OHCs}$ during late embryonic and perinatal ages (Lorenzen et al., 2015). We speculated that WT_P1 OHCs in cluster 1 originated from basal and middle turns as they more closely resembled WT_P7 and_P30 OHCs (Figure 6), whereas WT_P1 OHCs in cluster 4 that were grouped with the more immature E16 OHCs (Figure 6F,G) originated from apical turns.

Finally, trajectory analysis was applied to all the pooled cells (Figure $6 \mathrm{H}, \mathrm{I})$ ). Interestingly, two distinct tracks were observed: one reflected endogenous OHC development from E16 to P30 (black arrows in Figure $6 \mathrm{H}$ ); the other tracked the reprogramming process from adult SCs into $\mathrm{OHC}$ lineage (dotted arrows in Figure $6 \mathrm{H}$ ). Notably, in agreement with trajectory analysis described in Figure 6-figure supplement 3, OHC-like cells and nascent $\mathrm{HC}$ s were similar and intermingled with each other in both UMAP and trajectory analyses (Figure 6G,H).

\section{Neonatal-specific OHC genes are highly expressed in OHC-like cells}

We next took a more focused look at the transcriptomic differences and similarities between $\mathrm{OHC}$ like cells and WT_P1 OHCs. Based on two previous RNA-Seq studies (Kolla et al., 2020; Wiwatpanit et al., 2018), expression of 53 neonatal-specific OHC genes were further analyzed. These have been shown to be significantly enriched in WT_P0/P1 OHCs, but not in adult OHCs. The expression levels of these 53 genes in all $11 \mathrm{OHC}$-like cells was extracted from our raw data and expressed as transcripts per million (TPM). Those with TPM > 16 were defined as highly expressed genes; this threshold was chosen based on what gene expression level can typically be detected using RNA in situ hybridization with good signal to noise ratio in our hand. Our selection criteria for genes that were highly expressed in OHC-like cells thus were as follows: (1) that the gene's average TPM across all OHC-like cells was above 16; (2) that more than half of all OHC-like cells expressed this gene with TPM $>16$. If a gene did 
not meet either of the criteria, then it was classed as a gene differently expressed between OHC-like cells and WT_P1 OHCs.

Twenty-six of 53 (49.1\%) neonatal OHC genes, including Insm1 (red arrow in Figure 6-figure supplement 6A), were found to be highly expressed in OHC-like cells; $21 / 53(39.6 \%)$ of them, including $B c / 11 b, B m p 2$ and Bmp7, were expressed at low levels in these cells. Notably, Bcl11b is thought to be required for healthy stereocilia development (gray arrow in Figure 6-figure supplement 6A), as development of stereocilia in $\mathrm{OHCs}$ are defective in heterozygous $\mathrm{Bc} / 11 \mathrm{~b}^{ \pm}$mice (Okumura et al., 2011). Only one of the two criteria were met in $6 / 53$ (11.3\%) genes (green arrows in Figure 6-figure supplement 6A). The numerical TPM values of these categories of neonatal $\mathrm{OHC}$ genes in $\mathrm{OHC}$ like cells were included in Supplementary file 5. Together, based on the selected $53 \mathrm{OHC}$ genes analyzed, the overall similarity between all the $11 \mathrm{OHC}$-like cells and neonatal OHCs is $~ 50 \%$.

As OHC-like cells only highly expressed about half of WT_P1 OHCs characteristic genes, we asked whether there was any overlap with neonatal IHC or vestibular HC-specific genes. Akin to before, based on three previous studies (Burns et al., 2015; Kolla et al., 2020; Wiwatpanit et al., 2018), 49 neonatal IHC and 26 utricle HC characteristic genes were further analyzed (Figure 6figure supplement 6 B). The same two criteria as above were applied to dictate whether a gene was expressed at a high or low level in $\mathrm{OHC}$-like cells. None 0/49 (0\%) of neonatal IHC-specific genes were expressed at a high level in OHC-like cells. Among the 26 neonatal utricle HC-specific genes, 4/26 (35.4\%) were expressed at high levels in OHC-like cells, including F730043M19Rik, Cfap161, Nme9, and 1700001L19Rik (red arrows in Figure 6-figure supplement 6B). As an internal reference, we also included nine genes, including $5 / \mathrm{c} 26 \mathrm{a} 5$ and $\mathrm{Ocm}$, that were highly expressed in OHC-like cells (marked by red line in Figure 6-figure supplement 6B). The numerical TPM values of these neonatal $\mathrm{IHC}$ and utricle $\mathrm{HC}$-specific genes were included in Supplementary file 6. Taken together all the gene profiling results, we concluded that $\mathrm{OHC}$-like cells resemble neonatal $\mathrm{OHCs}$ and not neonatal IHCs or utricle HCs.

\section{OHC-like cells are far less differentiated than adult wild-type OHCs}

To complete our transcriptomic survey of $\mathrm{OHC}$-like cells, we compared their expression patterns to mature P30_WT OHCs (Figure 6-figure supplement 7A). In total, 1998 genes were significantly higher in OHC-like cells than in P30_WT OHCs. Of note, 67 \% (1339/1998) of these were overlapped with SC genes including Fgfr3, Id1, and Id2 (purple arrows in Figure 6-figure supplement 7A and Supplementary file 7), most likely due to the persistent expression of these SC genes that were not able to be downregulated in the converted $\mathrm{OHC}$-like cells. These remaining SC genes likely represented key barriers preventing $\mathrm{OHC}$-like cells from undergoing further maturation.

Conversely, 361 genes were expressed at a significantly lower level in OHC-like cells as compared to WT_P30 OHCs. Notably, $49.6 \%$ (179/361) of these were overlapped with OHC-enriched genes, including Slc26a5 (Prestin), Kcna10, and Lmo7 (green arrows in Figure 6-figure supplement 7A). Relevantly, Lmo7 mutant mice have been reported to show abnormalities in HC stereocilia (Du et al., 2019) and Kcna10 mutants exhibit mild auditory dysfunction (Lee et al., 2013). Thus, the relatively low expression of these functional proteins might account in part for the immature status of the new OHC-like cells. Supplementary file 7 contained the complete list of genes differently expressed genes between OHC-like cells and WT_P30 OHCs. GO analysis results showed that genes expressed at higher levels in $\mathrm{OHC}$-like cells were associated with cell adhesion (Figure 6-figure supplement 7B). The complete gene list associated with the GO cell adhesion category could be found in Supplementary file 8.

To conclude, the current differentiation or cell fate conversion status of the OHC-like cells was simply summarized in Figure 7, more manipulations are still needed to further promote maturation of $\mathrm{OHC}$-like cells. Decreasing the remaining SC genes and/or increasing $\mathrm{OHC}$ genes represent actionable and promising avenues.

\section{Discussion}

In summary, the merit of our study lies in the demonstration of effective conversion of adult cochlear SCs (mainly PCs and DCs) into Prestin+ $\mathrm{OHC}$-like cells after $\mathrm{OHC}$ damage in vivo. This experimental 


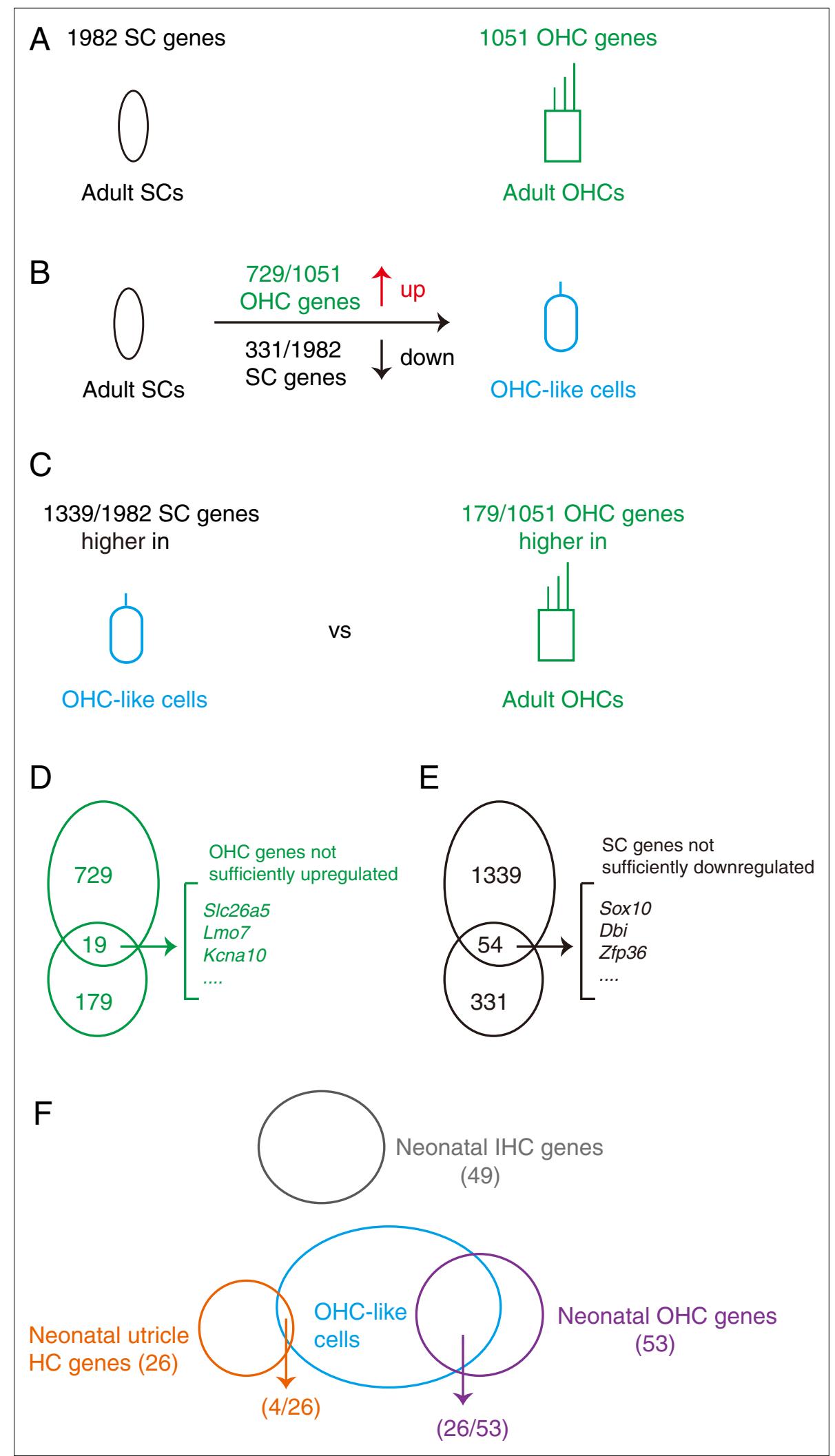

Figure 7. Summary of the differentiation status of the OHC-like cells. (A) Comparison of transcriptomic profiles revealed 1982 SC and 1,051 OHC differentially expressed genes. (B) Compared to adult SCs, OHC-like cells upregulated $729 \mathrm{OHC}$ genes and downregulated 331 SC genes, thus having become more OHC-like and less SC like. (C) Compared to adult OHCs, OHC-like cells expressed 1339 SC genes at higher levels, and $179 \mathrm{OHC}$ genes Figure 7 continued on next page 
Figure 7 continued

at lower levels, showing that $\mathrm{OHC}$ status had not yet been achieved. (D) $19 \mathrm{OHC}$ genes intersected between the upregulated $729 \mathrm{OHC}$ genes (as compared to adult SCs) and the under-expressed $179 \mathrm{OHC}$ genes (as compared to adult $\mathrm{OHCs}$ ), suggesting that although these genes were up-regulated in $\mathrm{OHC}$-like cells, their expression levels were still lower than that expected for adult OHCs. (E) Similar to (D), 54 SC genes overlapped between 1339 and 331 SC genes that were found to be at higher levels than expected for adult OHCs, but at lower levels than expected for adult SCs. These SC genes had thus successfully been downregulated, but not sufficiently so to reach the low levels in adult OHCs. (F) OHC-like cells expressed 26 of the 53 neonatal OHC genes, but only expressed 4 of the 26 neonatal utricle $\mathrm{HC}$ genes and did not express any neonatal IHC genes.

paradigm is clinically relevant and suggests that Atoh1 and Ikzf2 are candidate therapeutics for OHC regeneration.

\section{Potential roles of Atoh1 and Ikzf2 in cell-fate transition from adult cochlear SCs into OHC-like cells}

During normal HC development, Atoh1 is expressed for approximately 1 week, beginning at the basal end of the cochlea and progressively extending to the apex; expression is then down-regulated following the same temporal basal-to-apical sequence. During this period, Atoh1 is reported to be involved in at least three age-dependent processes: (1) specifying general HC fate, (2) maintaining the survival of $\mathrm{HC}$ progenitors (short term) and $\mathrm{OHCs}$ (long term), and (3) organizing the formation of hair bundles (Bermingham et alo, 1999; Cai et al., 2013; Woods et al., 2004). While Atoh1 is sufficient to convert neonatal PCs and DCs into HCs (Liu et alo, 2012a), it is insufficient to do so in adults. Ikzf2 alone also cannot convert adult SCs into HCs but the combination both Atoh 1 and Ikzf2 is synergistic and can do so in adults. Whether these effects are similar to the synergistic effects between Atoh1 and Pou4f3, which were recently reported during normal cochlear HC development (Yu et al., 2021), will require further investigation.

Ikzf2 is necessary for OHC maturation, and an Ikzf2 point-mutation model, Ikzf2 cello/cello, displays early-onset hearing loss and diminished Prestin expression; dual to this, ectopic lkzf2 induces Prestin expression in IHCs (Chessum et al., 2018). Whether Prestin is a direct target of Ikzf2 is unclear, but the onset of lkzf2 expression at neonatal ages and its specific and permanent expression in OHCs suggest that Ikzf2 is a key TF required to specify and maintain the $\mathrm{OHC}$ fate and/or repress the IHC fate. Therefore, we speculate that Ikzf2 was responsible for directing nascent $\mathrm{HCs}$ into the OHC differentiation track. In accord with this, Ikzf2 alone was able to induce Prestin expression in wild-type IHCs but not adult SCs, likely due to that IHCs are already in the general 'HC differentiation track' and thus more are poised to express $\mathrm{OHC}$ genes. In contrast to the ability of Atoh1, which takes approximately three weeks to convert SC to HC fate in the neonatal cochlea (Liu et al., 2012a), the combined effects of Atoh1 and Ikzf2 in mediating this cell-fate switch in the adult cochlea occurred within a shorter time window (approximately 12 days). This could be a result of the synergistic actions between Atoh 1 and Ikzf2.

\section{Active $\mathrm{OHC}$ death promotes transformation from adult SCs into OHC- like cells}

In our study, DT treatment led to selective $\mathrm{OHC}$ death, which was defined as active $\mathrm{OHC}$ death. In opposition to this, $\mathrm{OHC}$ death occurred as a side effect of overexpressing Ikzf2 alone or Ikzf2 and Atoh1 concurrently (Figure 2), which we refer to as passive OHC death. While the detailed mechanisms are unclear, passive $\mathrm{OHC}$ death has been observed in multiple instances upon: (1) overexpressing Atoh1 in neonatal or juvenile cochlear SCs (Liu et al., 2012a), (2) triggered neonatal SC proliferation (Liu et al., 2012b), and (3) conditional SC damage at postnatal ages (Mellado Lagarde et al., 2013; Mellado Lagarde et al., 2014). Our data showed that only OHC death that was 'active' could significantly increase numbers of OHC-like cells upon Atoh1 and lkzf2 overexpression in adult SCs (Figure 4I).

How to interpret the differential effects of active and passive OHC death? It is known that DT, but not neomycin, induced $\mathrm{HC}$ death can trigger Wnt-dependent $\mathrm{HC}$ regeneration from SCs in neonatal mice (Hu et al., 2016). This exemplifies that the precise way that $\mathrm{HC}$ dies can drastically affect how cochlear SCs respond to HC death. DT treatment causes cell membrane rupture leading to the release 
of cellular components in the extracellular space. We speculated that SCs overexpressing Atoh1 and Ikzf2 responded favorably to yet unidentified factors released from OHCs during active cell death. Such factors may not be made accessible to SCs upon passive OHC death.

We also conjectured that the timing of $\mathrm{OHC}$ active death in relation to the SC cellular status was likely the key. When active OHC death occurred by DT injection, Atoh1 and Ikzf2 had already been overexpressed in adult SCs for 6 days (Figure 4B). This particular temporal coupling may have permitted those adult $\mathrm{SCs}$ to respond more favorably to the unidentified factors released from nearby dying $\mathrm{OHCs}$, to ultimately become OHC-like cells. When no active death was induced, and instead $\mathrm{OHCs}$ died passively due to secondary effect of ectopic Atoh1 and lkzf2 expression, it may have been the timing between $\mathrm{SC}$ gene induction and $\mathrm{OHC}$ death that was unfavorable. Future studies will be needed to determine the main contributing factors dictating conversion efficiency or whether these hypothesis have any ground at all.

\section{Degree of cell fate conversion of the OHC-like cells}

By comparing the transcriptomic profiles between adult endogenous $\mathrm{OHCs}$ and $\mathrm{SCs}$, we identified 1051 OHC and 1982 SC genes, respectively (Figure 6-figure supplement 1A and Supplementary file 1). We then used the degree of $\mathrm{OHC}$ gene upregulation and $\mathrm{SC}$ gene downregulation to estimate the extent of cell fate conversion from adult SCs to OHC-like cells (Figure 7A). Promisingly, relative to adult SCs (primarily PCs and DCs), $69.4 \%$ (729/1051) of OHC genes were significantly up-regulated in OHC-like cells (Figure 7B). A more limited number of SC genes, 16.7 \% (331/1982), were significantly down-regulated in $\mathrm{OHC}$-like cells (Figure 7B). It appears that our reprogramming manipulations were more efficient at endowing $\mathrm{OHC}$-like status to SCs, than 'erasing/making them forget' their SC status.

To compare the transcriptomic profiles between $\mathrm{OHC}$-like cells and adult endogenous $\mathrm{OHCs}$, we selected endogenous $\mathrm{OHCs}$ at $\mathrm{P} 30$, but not $\mathrm{P} 60$, because $\mathrm{OHC}$-like cells were derived from SCs at P30 and thus their intrinsic age might be $\sim P 30$, although the mice were analyzed at P60. Nevertheless, cochlear development is complete by P30, and wild-type OHCs at P30 and P60 are expected to differ minimally. Consistently, it is reported that transcriptomic profiles of adult cochlear SCs at P60 and P120 are indistinguishable from each other (Hoa et alo, 2020). Thus, compared to adult endogenous OHCs, $17 \%$ (179/1051) of OHC genes were expressed at lower levels in OHC-like cells (Figure 7C). A substantial proportion of SC genes $67.6 \%(1339 / 1982)$ remained expressed at much higher level in $\mathrm{OHC}$-like cells than that in adult endogenous $\mathrm{OHCs}$ (Figure 7C). In addition, $19 \mathrm{OHC}$ genes, including Slc26a5, Lmo7, and Kcna10, were not sufficiently up-regulated in OHC-like cells (Figure 7D). Notably, $\mathrm{Ocm}$ was not included in the $19 \mathrm{OHC}$ genes, suggesting that $\mathrm{Ocm}$ was better upregulated than $\mathrm{Slc26a5}$ in the OHC-like cells. Likewise, $54 \mathrm{SC}$ genes, including Sox 10, Dbi, and Zfp36, were not sufficiently downregulated in OHC-like cells (Figure 7E).

In logic accord to only finding moderate transcriptomic similarities between $\mathrm{OHC}$-like cells and adult endogenous $\mathrm{OHCs}$, we identified stronger similarities with neonatal $\mathrm{OHCs}$ via different approaches (Figure 7F). Firstly, our integrated UMAP analysis embedded OHC-like cells closest to WT_P1 OHCs. Secondly, we found that OHC-like cells had upregulated 26 of the 53 known characteristic neonatal $\mathrm{OHC}$ genes. For instance, Insm1 was upregulated but not Bcl11b (Figure 6-figure supplement 6A). This was consistent with that Insm 1 is turned on earlier than $\mathrm{Bc} / 11 \mathrm{~b}$ during $\mathrm{OHC}$ development (Wiwatpanit et al., 2018).

We also investigated how similar OHC-like cells were to neonatal IHCs or utricle HCs. To do this, we first identified genes specifically enriched in neonatal IHCs or utricle HCs (Figure 6-figure supplement $6 \mathrm{~B}$ ) and extracted their expression level in $\mathrm{OHC}$-like cells. We found that neonatal IHC and utricle HC-specific genes were minimally expressed, if at all, in OHC-like cells. In addition, we noted that Cfap161, one of the four neonatal utricle HC genes expressed in OHC-like cells, is recently reported to be expressed in many cells with cilia structure including cochlear $\mathrm{HCs}$ via immunostaining assay (Beckers et al., 2021). Such discrepancies may be due to difference in the sensitivity of different techniques used. In addition, the current HC subtype specific genes were selected from three studies, but it is always challengeable and has limitations to compare data from different studies, especially the data are generated by different sequencing methods (Kolla et al., 2020). Nevertheless, more detailed gene profiling studies of different inner ear $\mathrm{HCs}$ at various developmental ages are needed to more precisely define some markers of individual $\mathrm{HC}$ subtypes. 
Despite many $\mathrm{OHC}$ genes were significantly upregulated, numerous SC genes were retained in the $\mathrm{OHC}$-like cells. Then, how best to define the cellular status of $\mathrm{OHC}$-like cells? Alternatively, are they more akin to OHCs or new hybrid OHCs/SCs taking a novel differentiation path? In our mind, there is no consensus yet to these questions, but great scope for exploration and discovery. We postulate that transcriptomic assays alone will likely be insufficient to define what constitutes a cell type for our purposes. Macro properties such as anatomical features, especially hair bundle structure in apical surface of HCs, as well as electrophysiological properties, will need to be measured. With that said, the most indisputable proof of an $\mathrm{OHC}$ cell state should be hearing recovery after $\mathrm{OHC}$ damage. In summary, our data supported that $\mathrm{OHC}$-like cells resemble neonatal $\mathrm{OHCs}$ and not neonatal IHCs or utricle HCs.

\section{Potential future approaches to promote more complete differentiation of OHC-like cells}

How to further promote differentiation of these OHC-like cells? This is the key question as the ultimate goal is to convert adult cochlear SCs into functional OHCs. To begin with, Atoh1 is only transiently expressed in cochlear OHCs during normal development (Cai et al., 2013; Driver et al., 2013; Matei et al., 2005). However, our model induced permanent Atoh1 expression, potentially hindering maturation of $\mathrm{OHC}$-like cells. Therefore, transient Atoh1 expression combined with permanent Ikzf2 expression may be the focus of future work.

In conjunction, we speculated that combining other candidate genes, together with Atoh1 and Ikzf2, would likely drive OHC-like cells to further mature. Promising candidate genes could include ones necessary for hair bundle organization and growth, as our results highlighted the reduced number and deformity of the stereocilia in $\mathrm{OHC}$-like cells (Figure 5). For instance, the neonatal OHC gene $\mathrm{Bcl11}$ b was missing in $\mathrm{OHC}$-like cells (gray arrows in Figure 6-figure supplement 6A) and heterozygous Bcl11 $b^{ \pm}$mice exhibit impaired formation of hair bundles in OHCs (Okumura et al., 2011). Simultaneous expression Atoh1, Ikzf2, and Bcl11b might therefore have higher reprogramming efficiency than Atoh1 and Ikzf2. Encouragingly, OHC-like cells did express multiple MET-channel-related proteins such as Cib2 and Tmc1 (Jia et al., 2020; Li et al., 2019; Pan et al., 2013). This aligned with the previous report that MET-channel assembly does not require normal hair bundle morphology (Cai et al., 2013).

As hair bundles mostly develop after birth, we also hypothesized that genes crucial for hair bundle organization are likely to be differentially expressed between P1 and P14/P30 OHCs. In terms of phenotypic assessment of functional hair bundles, rapid loss-of-function test will be performed following the protocols described in our previous pilot studies (Wang et al., 2021; Zhang et al., 2018). The phenotypes we hope to focus on will include shorter or disorganized OHC hair bundles, and ones with reduced numbers of $\mathrm{OHC}$ hair bundles at P14 or P30. Finally, we took note of the many SC genes expressed in OHC-like cells, including Fgfr3, Id1, and Id2 (Supplementary file 7). Fgf signaling is known to be involved in SC fate maintenance (Doetzlhofer et al., 2009; Jacques et al., 2007; Shim et al., 2005). Id gene family is known to repress HC formation by negatively regulating Atoh1 (Jones et al., 2006). Decreasing Fgfr3 and Id1/2 may promote further maturation of OHC-like cells.

In summary, our current study provided a genetic model for $\mathrm{OHC}$ damage and subsequent regeneration in adult mouse cochlea. Building on this model and adding further genetic manipulations represent a promising avenue to regenerate functional $\mathrm{OHCs}$.

\section{Materials and methods}

Key resources table

\begin{tabular}{lllll}
$\begin{array}{l}\text { Reagent type (species) } \\
\text { or resource }\end{array}$ & Designation & Source or reference & Identifiers & Additional information \\
\hline $\begin{array}{l}\text { Genetic reagent (Mus. } \\
\text { musculus) }\end{array}$ & FVB-Tg(Atoh1-cre/ERT)1Sbk/Mmnc MMRRC & Stock\#:029581-UNC & RRID:MMRRC_029581-UNC \\
\hline $\begin{array}{l}\text { Genetic reagent (Mus. } \\
\text { musculus) }\end{array}$ & Tg(Fgfr3-icre/ERT2)4-2Wdr & Jackson Lab & Stock\#:025809 & RRID:IMSR_JAX:025809
\end{tabular}

Continued on next page 


\section{Continued}

\begin{tabular}{|c|c|c|c|c|}
\hline $\begin{array}{l}\text { Reagent type (species) } \\
\text { or resource }\end{array}$ & Designation & Source or reference & Identifiers & Additional information \\
\hline $\begin{array}{l}\text { Genetic reagent (Mus. } \\
\text { musculus) }\end{array}$ & Ai9 & Jackson Lab & Stock\#:007909 & RRID:IMSR_JAX:007909 \\
\hline $\begin{array}{l}\text { Genetic reagent (Mus. } \\
\text { musculus) }\end{array}$ & Slc $26 a 5^{\mathrm{CreER/+}}$ & & Fang et al., 2012 & PMID:21954035 \\
\hline $\begin{array}{l}\text { Genetic reagent (Mus. } \\
\text { musculus) }\end{array}$ & CAG-LSL-Atoh1+ & & Liu et al., 2012a & PMID:22573682 \\
\hline $\begin{array}{l}\text { Genetic reagent (Mus. } \\
\text { musculus) }\end{array}$ & Rosa26 $6^{\text {CAG-LSL-kff2/+ }}$ & Jackson Lab & Stock\#:036272 & $\begin{array}{l}\text { Knock-in mouse line that will } \\
\text { be available soon }\end{array}$ \\
\hline $\begin{array}{l}\text { Genetic reagent (Mus. } \\
\text { musculus) }\end{array}$ & Slc26a $5^{\text {DTR/+ }}$ & Jackson Lab & Stock\#:036271 & $\begin{array}{l}\text { Knock-in mouse line that will } \\
\text { be available soon }\end{array}$ \\
\hline Antibody & anti-HA (Rat monoclonal) & Roche & Cat.\#:11867423001 & RRID:AB_390918 \\
\hline Antibody & anti-Prestin (Goat polyclonal) & Santa Cruz & Cat.\#:sc-22692 & RRID:AB_2302038 \\
\hline Antibody & anti-Myosin-VI (Rabbit polyclonal) & Proteus Bioscience & Cat.\#:25-6791 & RRID:AB_2314836 \\
\hline Antibody & anti-Myosin-VIla (Rabbit polyclonal) & Proteus Bioscience & Cat.\#:25-6790 & RRID:AB_10015251 \\
\hline Antibody & anti-Insm1 (Guinea pig) & $\begin{array}{l}\text { A kind gift from Dr. Shiqi Jia } \\
\text { and Dr. Carmen Birchmeier }\end{array}$ & Jia et al., 2015 & PMID:25828096 \\
\hline Antibody & $\begin{array}{l}\text { anti-Parvalbumin (Mouse } \\
\text { monoclonal) }\end{array}$ & Sigma-Aldrich & Cat.\#:P3088 & RRID:AB_477329 \\
\hline Antibody & anti-Rbm24 (Rabbit polyclonal) & Proteintech & Cat.\#:18178-1-AP & RRID:AB_2878513 \\
\hline Antibody & anti-Calbindin (Rabbit monoclonal) & Sigma-Aldrich & Cat.\#:C9848 & RRID:AB_2314067 \\
\hline Antibody & anti-vGlut3 (Rabbit polyclonal) & Synaptic System & Cat.\#:135,203 & RRID:AB_887886 \\
\hline Antibody & anti-Ocm (Rabbit) & Swant & Cat.\#:OMG4 & RRID:AB_10000346 \\
\hline Antibody & anti-Slc7a14 (Rabbit polyclonal) & Sigma-Aldrich & Cat.\#:HPA045929 & RRID:AB_2679501 \\
\hline Antibody & $\begin{array}{l}\text { anti-Ctbp2 } \\
\text { (Mouse monoclonal) }\end{array}$ & BD Biosciences & Cat.\#:612,044 & RRID:AB_399431 \\
\hline Commercial assay, kit & SMART-Seq HT Kit & Takara & Cat.\#:634,437 & $\begin{array}{l}\text { For reverse-transcription and } \\
\text { cDNA amplification }\end{array}$ \\
\hline Commercial assay, kit & $\begin{array}{l}\text { TruePrep DNA Library Prep Kit V2 } \\
\text { for Illumina }\end{array}$ & Vazyme & Cat.\#:TD503-02 & $\begin{array}{l}\text { For sequencing library } \\
\text { construction }\end{array}$ \\
\hline Commercial assay, kit & TruePrep Index Kit V2 for Illumina & Vazyme & Cat.\#:TD202 & $\begin{array}{l}\text { For sequencing library } \\
\text { construction (index) }\end{array}$ \\
\hline
\end{tabular}

\section{Generation of Rosa26 $6^{\text {CAG-LSL-lkzf2/+ }}$ and Slc26a5 $5^{\mathrm{DTR} /+}$ knock-in mouse strains using CRISPR/Cas9}

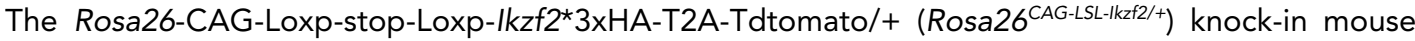
strain was produced by co-injecting one sgRNA against the Rosa26 locus (5'-actccagtctttctagaaga-3'), donor DNA (Figure 1-figure supplement 1), and Cas9 mRNA into one-cell-stage mouse zygotes. A similar approach was followed to generate the Slc26a5-P2A-DTR/+ (Slc26a5 $\left.{ }^{\text {DTR/+}}\right)$ knock-in mouse line. In this case, sgRNA targeting the Slc26a5 (Prestin) locus was used: 5'-CGAGGCATAAAGGCCCTGTA-3'. Information regarding the donor DNA is described in Figure 3-figure supplement 1. F0 mice of both strains were screened for potential successful insertion by performing junction PCR; this was followed by crossing the positive F0 mice with wild-type C57BL/6 mice to assess the germ-line status and to produce stable F1 mice. F1 mice was further confirmed via junction PCR. Moreover, lack of random insertion of donor DNA in the genome of these F1 mice was confirmed using Southern blotting (Figure 1-figure supplement 1D,E and Figure 3-figure supplement 1D,E), which was performed according to our previously published protocol (Li et al., 2018b). Both mouse strains were PCR-genotyped using tail DNA (representative gel images of PCR products were presented in Figure 1-figure supplement $1 F$ and Figure 3-figure supplement 1F). Details regarding primer 
sequences can be found in Supplementary file 9. All mice were bred and raised in SPF-level animal rooms, and animal procedures were performed according to the guidelines (NA-032-2019) of the IACUC of Institute of Neuroscience (ION), CAS Center for Excellence in Brain Science and Intelligence Technology, Chinese Academy of Sciences.

\section{Sample processing, histology, and immunofluorescence assays, and cell counting}

Adult mice were anesthetized and sacrificed during which hearts were perfused with $1 \times$ phosphatebuffered saline (PBS) and fresh $4 \%$ paraformaldehyde (PFA) to completely remove blood from the inner ear. Inner ear tissues were then carefully dissected out, re-fixed with fresh $4 \%$ PFA overnight at $4{ }^{\circ} \mathrm{C}$, and washed thrice with $1 \times$ PBS. The tissues were decalcified first with $120 \mathrm{mM}$ EDTA for 2 days at $4{ }^{\circ} \mathrm{C}$ until they were soft enough for micro-dissection and whole-mount preparation. Immunostaining was then performed with the following primary antibodies: anti-HA (rat, 1:200, 11867423001, Roche, RRID:AB_390918), anti-Prestin (goat, 1:1000, sc-22692, Santa Cruz, RRID:AB_2302038), anti-Myo6 (rabbit, 1:500, 25-6791, Proteus Bioscience, RRID:AB_2314836), antiMyo7a (rabbit, 1:500, 25-6790, Proteus Bioscience, RRID:AB_10015251), anti-Ocm (rabbit, 1:500, OMG-4, Swant, RRID:AB_10000346), anti-Insm1 (guinea pig, 1:6000; a kind gift from Dr. Shiqi Jia from Jinan University, Guangzhou, China, and Dr. Carmen Birchmeier from Max Delbrück Center for Molecular Medicine, Berlin, Germany) (Jia et al., 2015), anti-Ctbp2 (mouse, 1:500, 612044, BD Bioscience, RRID:AB_399431), anti-Parvalbumin (mouse, 1:500, P3088, Sigma-Aldrich, RRID:AB_477329), anti-Rbm24 (rabbit, 1:500, 18178-1-AP, Proteintech, RRID:AB_2878513), anti-Slc7a14 (rabbit, 1:500, HPA045929, Sigma-Aldrich, RRID:AB_2679501), anti-Calbindin (rabbit, 1:500, C9848, Sigma-Aldrich, RRID:AB_2314067), and anti-vGlut3 (rabbit, 1:500, 135203, Synaptic System, RRID:AB_887886). Cochlear tissues were counterstained with Hoechst 33,342 solution (1:1000, 62249, Thermo Scientific, RRID:AB_2651135) to visualize nuclei, and were mounted with Prolong gold antifade medium (P36930, Thermo Scientific, RRID: SCR_015961). Nikon C2, TiE-A1, and NiE-A1 plus confocal microscopes were used to capture images.

Each whole-mount preparation of individual cochlear duct was divided into three parts and imaged at 10x magnification using a confocal microscope. In each acquired image, a line was drawn passing through the center of the $\mathrm{IHCs}$ and $\mathrm{OHCs}$ to measure the full length of each cochlear duct. They were then divided into basal, middle, and apical portions of equal length. To assess the extent of $\mathrm{OHC}$ death in the Slc26a5 $5^{\mathrm{DTR} /+}$ model (Figure 3), the ratio of the $\mathrm{OHC}$ to IHC numbers was used as a proxy as IHC were not affected by DT treatment. To this end, $\mathrm{OHC}$ and IHC numbers within a scanning region $(60 \times$, confocal microscopy) were computed. This was repeated for two areas in each cochlear turn and mean number was calculated. To count Prestin+ IHCs in Atoh1-CreER+; Rosa26 CAGLSL-lkzf2/+ mice (Figure 1D), and Tdtomato+/HA+ cells, OHC-like cells, and nascent HCs in Fgfr3-Atoh1Ikzf2 and Fgfr3-Atoh1-lkzf2-DTR mice (Figures 2 and 4), the entire cochlear duct of each mouse was scanned to minimize variation between different replicates (60 ×, confocal microscope) and cells were counted manually. All cell counting data were presented as means \pm SEM. Statistical analyses were performed using one-way ANOVA, followed by a Student's t-test with Bonferroni correction. All statical analyses were implemented using GraphPad Prism 6.0.

\section{Single-cell RNA sequencing and bioinformatic analysis}

Single-cell RNA sequencing was performed in three different mouse lines; each time the cells of interest were identifiable by Tdtomato expression. Cells originated: (1) from P30 Slc26a5 ${ }^{\mathrm{CreER} /+}$; Ai9/+ mice that were injected with tamoxifen at P20 and P21; in this case, Tdtomato+ cells were exclusively $\mathrm{OHCs}$ due to selective Cre expression in OHCs (Fang et al., 2012). (2) from P60 Fgfr3-iCreER+; Ai9/+ mice that were administered tamoxifen at P30 and P31; in this instance, Tdtomato+ cells within the cochlear sensory epithelium were SCs (primarily PCs and DCs) according to our previous reports (Liu et al., 2012a; Liu et al., 2012b). (3) from P60 Fgfr3-Atoh1-lkzf2-DTR mice that were administered tamoxifen at P30 and P31 and then DT at P36. In this case, Tdtomato+ cells within the cochlear sensory epithelium included $\mathrm{OHC}$-like cells, nascent $\mathrm{HCs}$, and SCs failing to become HCs.

The cochlear sensory epithelium from each mouse was dissected out, digested, and single-cell suspensions were prepared according to our previously described protocol (Li et al., 2020b). All the aforementioned Tdtomato+ cells were picked under a fluorescence microscope (M205FA, Leica) as 
illustraed in Figure 6A. We picked 17 wild-type adult OHCs at P30, 16 wild-type adult SCs at P60, and 42 Tdtomato+ cells from the Fgfr3-Atoh1-Ikzf2-DTR model at P60 (Figure 6D). Once the cells were picked, they were immediately subjected to reverse-transcription and cDNA amplification using a Smart-Seq HT kit (Cat\# 634437, Takara). The cDNAs (1 ng each) were tagmented using a TruePrep DNA Library Prep Kit V2 for Illumina (Cat\# TD503-02, Vazyme) and a TruePrep Index Kit V2 for Illumina (Cat\# TD202, Vazyme). The final libraries were subject to paired-end sequencing on the Illumina Novaseq platform. Each library was sequenced leading to $4 \mathrm{G}$ of raw data.

FastQC (v0.11.9) and trimmomatic (v0.39) were used for quality control of raw sequencing data. High-quality mapping to the mouse reference genome (GRCm38) was achieved for $70-80 \%$ of the reads using the Hisat2 (v2.1.0) program with default parameters. Raw counts were calculated using HTSeq (v0.10.0), and gene-expression levels were estimated by using StringTie (v1.3.5) with default parameters. Gene abundances were presented as transcript per million (TPM) values. Differentially expressed genes (DEGs) were analyzed using R package 'DESeq2' $(p<0.001$, absolute value of (log2 fold change) $>2$ ). Based on the identified DEGs, biological process enrichment was evaluated $(p<0.001$, adjusted using FDR correction) by using DAVID (Database for Annotation, Visualization and Integrated Discovery). Extensive quality checks of the data were carried out throughout the analyses. Detailed information regarding mapping rate to mouse genome, neumbers of detected genes with TPM $>1$ and TPM > 10, as well as cell names and their corresponding cell identities could be found in Supplementary file 10. The raw data generated from this single-cell RNA sequencing study is accessibly in the GEO (Gene Expression Omnibus) database under Accession No. GSE161156.

To analyze the previously published RNA-seq data of wild-type OHCs at E16, P1, and P7 in a recent study (Kolla et al., 2020), the Seurat processing pipeline (R package v3.0) was applied. To adequately compare the transcriptomic profiles obtained from 10× genomics and smartseq methods, we first integrated them by using the Seurat functions 'SCTransform', 'FindlntegrationAnchors' (k.filter $=30$ ), and 'IntegrateData'. Principal component analysis (PCA) was performed by executing the 'RunPCA' function, and the top 20 principal components (PCs) were used for further dimensionality reduction using UMAP (via 'RunUMAP'). Unsupervised clustering was performed using the 'FindNeighbors' and 'FindClusters' function (resolution $=0.5$ for Figure 6 -figure supplement 5A, 0.8 for Figure 6F, or 1.5 for Figure 6D).

Furthermore, E16_WT OHCs were defined as cells in which the expression level of Insm1, Myo6, and Atoh1 were all above zero; P1_WT OHCs were identified as those with above zero expression of Bcl11b, Myo6, Myo7a, and Atoh1. Finally, cells expressing above zero level of Slc26a5 (Prestin), Myo6, $\mathrm{Ocm}$, and Ikzf2 were classified as P7_WT OHCs. Trajectory analysis was performed using Monocle ( $R$ package v2.0). Pre-processed Seurat datasets were imported into Monocle. The top 2000 most variable genes identified from the Seurat object were used as data input to Monocle. Cells were ordered along a pseudotime axis by using the 'orderCells' function in Monocle. The pseudotime scale in monocle is an arbitrary unit, and it is simulated according to the developmental order (or age/ differentiation status) of each cell.

\section{ABR measurement}

$A B R$ were measured in response to the following sound frequencies: $4 k, 5.6 k, 8 k, 11.3 k, 16 k, 22.6 k$, and $32 \mathrm{k} \mathrm{Hz}$, following our previously published (Li et al., 2018b). To assess statistical significance between responses of the same frequency between conditions, Student's t-tests were performed (Figures 3C and 5D).

\section{Tamoxifen and DT treatment}

Tamoxifen (Cat\# T5648, Sigma-Aldrich) was dissolved in corn oil (Cat\# C8267, Sigma-Aldrich) and injected intraperitoneally at $3 \mathrm{mg} / 40 \mathrm{~g}$ body weight (for P0 and P1 mice) or at $9 \mathrm{mg} / 40 \mathrm{~g}$ body weight (for P20 and P21, and P30 and P31 mice). DT (Cat\# D0564, Sigma-Aldrich) dissolved in $0.9 \% \mathrm{NaCl}$ solution was also delivered through intraperitoneal injection, at a dose of $20 \mathrm{ng} / \mathrm{g}$ body weight. The mice were sacrificed at P42, P46, or P60.

\section{SEM data collection and analysis}

SEM was performed following the protocol reported previously (Parker et al., 2016). Briefly, we made holes at the cochlear apex. Cochlear samples were then washed gently with $0.9 \% \mathrm{NaCl}$ 
(Cat\#10019318, Sinopharm Chemical Reagent Co, Ltd.) and fixed overnight with $2.5 \%$ glutaraldehyde (Cat\# G5882, Sigma-Aldrich) at $4{ }^{\circ} \mathrm{C}$. On the following day, the cochlear samples were washed thrice with $1 \times$ PBS and subsequently decalcified using $10 \%$ EDTA (Cat\# ST066, Beyotime) for 1 day, then refixed for $1 \mathrm{~h}$ with $1 \%$ osmium tetroxide (Cat\#18451, Ted Pella), and subject to a second fixation with thiocarbohydrazide (Cat\#88535, Sigma-Aldrich) for $30 \mathrm{~min}$ and further fixed fixation for $1 \mathrm{hr}$ with osmium tetroxide. Next, the cochlear samples were dehydrated using a graded ethanol series (30\%, 50\%, 75\%, 80\%, $95 \%$; Cat\#10009259, Sinopharm Chemical Reagent Co, Ltd.) at $4{ }^{\circ} \mathrm{C}$ with each incubation step lasting $30 \mathrm{~min}$. The series was completed by fully dehydrating the samples in $100 \%$ ethanol (three times, $30 \mathrm{~min}$ for each) at $4{ }^{\circ} \mathrm{C}$. The cochlear samples were then dried in a critical point dryer (Model: EM CPD300, Leica), after which whole-mount cochlear samples were prepared under a microscope to ensure that hair bundles were facing upward. Finally, samples were treated with a turbomolecular pumped coater (Model: Q150T ES, Quorum). The prepared samples were then scanned using a field-emission SEM instrument (Model: GeminiSEM 300, ZEISS).

\section{Acknowledgements}

We thank Drs. Qian Hu, Yu Kong, and Xu Wang from Optical Imaging and EM Facility of the Institute of Neuroscience (ION) for support with the image analysis; Dr. Hui Yang (Principal Investigator at the ION) for sharing the zygote microinjection system used to generate the knockin mice; Ms. Qian Liu (from the Department of Embryology of the ION animal center) for helping us in transplanting zygotes into pseudopregnant female mice; Dr. Shiqi Jia (Jinan University, Guangzhou, China) and Dr. Carmen Birchmeier (Max Delbrück Center for Molecular Medicine, Berlin, Germany) for kindly providing the anti-Insm1 antibody; and Ms.Virginia M S Rutten (HHMI-Janelia Research Campus, Ashburn, VA, USA) for assistance in editing the text.

\section{Additional information}

\section{Competing interests}

Suhong Sun, Shuting Li, Zhengnan Luo, Minhui Ren, Shunji He, Guangqin Wang, Zhiyong Liu: We filed an auditory hair cell regeneration patent based on the key findings of this manuscript..

Funding

\begin{tabular}{|c|c|c|}
\hline Funder & Grant reference number & Author \\
\hline $\begin{array}{l}\text { National Natural Science } \\
\text { Foundation of China }\end{array}$ & 81771012 & Zhiyong Liu \\
\hline $\begin{array}{l}\text { Ministry of Science and } \\
\text { Technology of the People's } \\
\text { Republic of China }\end{array}$ & 2017YFA0103901 & Zhiyong Liu \\
\hline $\begin{array}{l}\text { Chinese Academy of } \\
\text { Sciences }\end{array}$ & XDB32060100 & Zhiyong Liu \\
\hline $\begin{array}{l}\text { Shanghai Municipal Bureau } \\
\text { of Quality and Technical } \\
\text { Supervision }\end{array}$ & 2018SHZDZX05 & Zhiyong Liu \\
\hline $\begin{array}{l}\text { Shanghai Jiao Tong } \\
\text { University }\end{array}$ & SSMU-ZLCX20180601 & Zhiyong Liu \\
\hline Boehringer Ingelheim & DE811138149 & Zhiyong Liu \\
\hline
\end{tabular}

The funders had no role in study design, data collection and interpretation, or the decision to submit the work for publication.

Author contributions

Suhong Sun, Zhengnan Luo, Data curation, Formal analysis, Writing - review and editing; Shuting Li, Data curation, Formal analysis, Investigation, Writing - review and editing; Minhui Ren, Formal analysis, Software; Shunji He, Guangqin Wang, Formal analysis, Methodology; Zhiyong Liu, Conceptualization, 
Formal analysis, Funding acquisition, Investigation, Project administration, Supervision, Writing - original draft, Writing - review and editing

Author ORCIDs

Suhong Sun (iD) http://orcid.org/0000-0003-0059-4534

Shuting Li (iD) http://orcid.org/0000-0003-3438-1588

Zhengnan Luo (D) http://orcid.org/0000-0002-8204-6277

Zhiyong Liu (iD) http://orcid.org/0000-0002-9675-1233

Ethics

All mice were bred and raised in SPF level animal rooms and animal procedures were performed according to guidelines (NA-032-2019) of the IACUC of Institute of Neuroscience (ION), Chinese Academy of Sciences.

Decision letter and Author response

Decision letter https://doi.org/10.7554/eLife.66547.sa1

Author response https://doi.org/10.7554/eLife.66547.sa2

Additional files

Supplementary files

- Supplementary file 1. List of all genes that were expressed at higher $(P<0.001)$ levels in WT_P30 OHCs as compared to WT_P60 SCs, and vice versa. The most differentially expressed genes were listed in Figure 6-figure supplement $1 \mathrm{~A}$.

- Supplementary file 2. GO analysis of genes that were significantly enriched in WT_P30 OHCs, relative to WT_P60 SCs. Top GO terms were shown in Figure 6-figure supplement 1B.

- Supplementary file 3. GO analysis of genes that were significantly enriched in OHC-like cells, relative to WT_P60 SCs. Top GO terms were presented in Figure 6-figure supplement 2B.

- Supplementary file 4. List of genes expressed at higher level in OHC-like cells relative to WT_P60 SCs, and vice versa. In addition, these genes which were overlapped with $\mathrm{OHC}$ or SC-enriched genes (Figure 6-figure supplement 2A) are also included in a separate tab in the file.

- Supplementary file 5. TPM values in $\mathrm{OHC}$-like cells of 53 neonatal $\mathrm{OHC}$ genes, as presented in Figure 6-figure supplement 6A.

- Supplementary file 6. TPM values in $\mathrm{OHC}$-like cells of 9 pan-HC or OHC-enriched genes, 49 neonatal IHC genes, and 26 neonatal utricle $\mathrm{HC}$ genes, as presented in Figure 6-figure supplement 6B.

- Supplementary file 7. List of all genes that were differently expressed between $\mathrm{OHC}$-like cells and WT_P30 OHCs. In addition, we also included those differently expressed genes that were overlapped with SC or OHC genes (presented in Figure 6-figure supplement 7A), as well as the $19 \mathrm{OHC}$ genes (not sufficiently upregulated) or 54 SC genes (not sufficiently downregulated) in $\mathrm{OHC}$-like cells.

- Supplementary file 8. GO analysis of genes that were significantly enriched in OHC-like cells relative to WT_P30 OHCs. The results were presented in Figure 6-figure supplement 7B.

- Supplementary file 9. Genotyping primers, and sizes of PCR amplicons for the various knock-in and transgenic mouse strains used in this study.

- Supplementary file 10. Detailed information of all the 75 single cells that were subject to smartseq analysis. Notably, the OHC-like cells, nascent HCs and SCs failing to become HCs were picked from the same model and labelled exp-1 to exp-42 in the file. Their cell identity was defined after bioinformatic analysis.

- Transparent reporting form

Data availability

Sequencing data have been deposited in GEO under accession codes: GSE161156. 
The following previously published datasets were used:

\begin{tabular}{|c|c|c|c|c|}
\hline Author(s) & Year & Dataset title & Dataset URL & Database and Identifier \\
\hline Kolla & 2020 & $\begin{array}{l}\text { Characterization of } \\
\text { cochlear development at } \\
\text { the single cell level }\end{array}$ & $\begin{array}{l}\text { https://www.ncbi. } \\
\text { nlm.nih.gov/geo/ } \\
\text { query/acc.cgi?acc= } \\
\text { GSE137299 }\end{array}$ & $\begin{array}{l}\text { NCBI Gene Expression } \\
\text { Omnibus, GSE137299 }\end{array}$ \\
\hline
\end{tabular}

\section{References}

Atkinson PJ, Huarcaya Najarro E, Sayyid ZN, Cheng AG. 2015. Sensory hair cell development and regeneration: similarities and differences. Development 142: 1561-1571. DOI: https://doi.org/10.1242/dev.114926, PMID: 25922522

Beckers A, Fuhl F, Ott T, Boldt K, Brislinger MM, Walentek P, Schuster-Gossler K, Hegermann J, Alten L, Kremmer E. 2021. The highly conserved FOXJ1 target CFAP161 is dispensable for motile ciliary function in mouse and Xenopus. Scientific Reports 11: 13333. DOI: https://doi.org/10.1038/s41598-021-92495-3, PMID: 34172766

Bermingham NA, Hassan BA, Price SD, Vollrath MA, Ben-Arie N, Eatock RA, Bellen HJ, Lysakowski A, Zoghbi HY. 1999. Math1: an essential gene for the generation of inner ear hair cells. Science 284: 1837-1841. DOI: https:// doi.org/10.1126/science.284.5421.1837, PMID: 10364557

Buran BN, Strenzke N, Neef A, Gundelfinger ED, Moser T, Liberman MC. 2010. Onset coding is degraded in auditory nerve fibers from mutant mice lacking synaptic ribbons. The Journal of Neuroscience 30: 7587-7597. DOI: https://doi.org/10.1523/JNEUROSCI.0389-10.2010, PMID: 20519533

Burns JC, Kelly MC, Hoa M, Morell RJ, Kelley MW. 2015. Single-cell RNA-Seq resolves cellular complexity in sensory organs from the neonatal inner ear. Nature Communications 6: 8557. DOI: https://doi.org/10.1038/ ncomms9557, PMID: 26469390

Cai T, Seymour ML, Zhang H, Pereira FA, Groves AK. 2013. Conditional deletion of Atoh1 reveals distinct critical periods for survival and function of hair cells in the organ of Corti. The Journal of Neuroscience 33: 1011010122. DOI: https://doi.org/10.1523/JNEUROSCl.5606-12.2013, PMID: 23761906

Chai R, Kuo B, Wang T, Liaw EJ, Xia A, Jan TA, Liu Z, Taketo MM, Oghalai JS, Nusse R. 2012. Wnt signaling induces proliferation of sensory precursors in the postnatal mouse cochlea. PNAS 109: 8167-8172. DOI: https://doi.org/10.1073/pnas.1202774109, PMID: 22562792

Chen Y, Gu Y, Li Y, Li GL, Chai R, Li W, Li H. 2021. Generation of mature and functional hair cells by co-expression of Gfi1, Pou4f3, and Atoh1 in the postnatal mouse cochlea. Cell Reports 35: 109016. DOI: https://doi.org/10. 1016/j.celrep.2021.109016

Chessum L, Matern MS, Kelly MC, Johnson SL, Ogawa Y, Milon B, McMurray M, Driver EC, Parker A, Song Y. 2018. Helios is a key transcriptional regulator of outer hair cell maturation. Nature 563: 696-700. DOI: https:// doi.org/10.1038/s41586-018-0728-4, PMID: 30464345

Chow LM, Tian Y, Weber T, Corbett M, Zuo J, Baker SJ. 2006. Inducible Cre recombinase activity in mouse cerebellar granule cell precursors and inner ear hair cells. Developmental Dynamics 235: 2991-2998. DOI: https://doi.org/10.1002/dvdy.20948, PMID: 16958097

Corey DP, Holt JR. 2016. Are TMCs the Mechanotransduction Channels of Vertebrate Hair Cells. The Journal of Neuroscience 36: 10921-10926. DOI: https://doi.org/10.1523/JNEUROSCI.1148-16.2016, PMID: 27798174

Costa A, Sanchez-Guardado L, Juniat S, Gale JE, Daudet N, Henrique D. 2015. Generation of sensory hair cells by genetic programming with a combination of transcription factors. Development 142: 1948-1959. DOI: https://doi.org/10.1242/dev.119149, PMID: 26015538

Cox BC, Liu Z, Lagarde MM, Zuo J. 2012. Conditional gene expression in the mouse inner ear using Cre-loxP. Journal of the Association for Research in Otolaryngology 13: 295-322. DOI: https://doi.org/10.1007/ s10162-012-0324-5, PMID: 22526732

Cox BC, Chai R, Lenoir A, Liu Z, Zhang L, Nguyen DH, Chalasani K, Steigelman KA, Fang J, Rubel EW. 2014. Spontaneous hair cell regeneration in the neonatal mouse cochlea in vivo. Development 141: 816-829. DOl: https://doi.org/10.1242/dev.103036, PMID: 24496619

Doetzlhofer A, Basch ML, Ohyama T, Gessler M, Groves AK, Segil N. 2009. Hey2 regulation by FGF provides a Notch-independent mechanism for maintaining pillar cell fate in the organ of Corti. Developmental Cell 16: 58-69. DOI: https://doi.org/10.1016/j.devcel.2008.11.008, PMID: 19154718

Douguet D, Honore E. 2019. Mammalian Mechanoelectrical Transduction: Structure and Function of ForceGated Ion Channels. Cell 179: 340-354. DOI: https://doi.org/10.1016/j.cell.2019.08.049, PMID: 31585078

Driver EC, Sillers L, Coate TM, Rose MF, Kelley MW. 2013. The Atoh1-lineage gives rise to hair cells and supporting cells within the mammalian cochlea. Developmental Biology 376: 86-98. DOI: https://doi.org/10. 1016/j.ydbio.2013.01.005, PMID: 23318633

Du TT, Dewey JB, Wagner EL, Cui R, Heo J, Park JJ, Francis SP, Perez-Reyes E, Guillot SJ, Sherman NE. 2019. LMO7 deficiency reveals the significance of the cuticular plate for hearing function. Nature Communications 10: 1117. DOI: https://doi.org/10.1038/s41467-019-09074-4, PMID: 30850599

Fang J, Zhang WC, Yamashita T, Gao J, Zhu MS, Zuo J. 2012. Outer hair cell-specific prestin-CreERT2 knockin mouse lines. Genesis 50: 124-131. DOI: https://doi.org/10.1002/dvg.20810, PMID: 21954035 
Giese APJ, Tang YQ, Sinha GP, Bowl MR, Goldring AC, Parker A, Freeman MJ, Brown SDM, Riazuddin S, Fettiplace R. 2017. CIB2 interacts with TMC1 and TMC2 and is essential for mechanotransduction in auditory hair cells. Nature Communications 8: 43. DOI: https://doi.org/10.1038/s41467-017-00061-1, PMID: 28663585

Golub JS, Tong L, Ngyuen TB, Hume CR, Palmiter RD, Rubel EW, Stone JS. 2012. Hair cell replacement in adult mouse utricles after targeted ablation of hair cells with diphtheria toxin. The Journal of Neuroscience 32: 15093-15105. DOI: https://doi.org/10.1523/JNEUROSCI.1709-12.2012, PMID: 23100430

Grifone R, Saquet A, Xu Z, Shi DL. 2018. Expression patterns of Rbm24 in lens, nasal epithelium, and inner ear during mouse embryonic development. Developmental Dynamics 247: 1160-1169. DOI: https://doi.org/10. 1002/dvdy.24666, PMID: 30133047

Groves AK, Zhang KD, Fekete DM. 2013. The genetics of hair cell development and regeneration. Annual Review of Neuroscience 36: 361-381. DOI: https://doi.org/10.1146/annurev-neuro-062012-170309, PMID: 23724999

Hoa M, Olszewski R, Li X, Taukulis I, Gu S, DeTorres A, Lopez IA, Linthicum FH, Ishiyama A, Martin D. 2020. Characterizing Adult Cochlear Supporting Cell Transcriptional Diversity Using Single-Cell RNA-Seq: Validation in the Adult Mouse and Translational Implications for the Adult Human Cochlea. Frontiers in Molecular Neuroscience 13: 13. DOI: https://doi.org/10.3389/fnmol.2020.00013, PMID: 32116546

Hu L, Lu J, Chiang H, Wu H, Edge AS, Shi F. 2016. Diphtheria Toxin-Induced Cell Death Triggers Wnt-Dependent Hair Cell Regeneration in Neonatal Mice. The Journal of Neuroscience 36: 9479-9489. DOI: https://doi.org/10. 1523/JNEUROSCI.2447-15.2016, PMID: 27605621

Jacques BE, Montcouquiol ME, Layman EM, Lewandoski M, Kelley MW. 2007. Fgf8 induces pillar cell fate and regulates cellular patterning in the mammalian cochlea. Development 134: 3021-3029. DOI: https://doi.org/ 10.1242/dev.02874, PMID: 17634195

Janesick AS, Heller S. 2019. Stem Cells and the Bird Cochlea-Where Is Everybody. Cold Spring Harbor Perspectives in Medicine 9: a033183. DOI: https://doi.org/10.1101/cshperspect.a033183, PMID: 30249599

Jeng JY, Ceriani F, Hendry A, Johnson SL, Yen P, Simmons DD, Kros CJ, Marcotti W. 2020. Hair cell maturation is differentially regulated along the tonotopic axis of the mammalian cochlea. The Journal of Physiology 598: 151-170. DOI: https://doi.org/10.1113/JP279012, PMID: 31661723

Jia S, Ivanov A, Blasevic D, Muller T, Purfurst B, Sun W, Chen W, Poy MN, Rajewsky N, Birchmeier C. 2015. Insm1 cooperates with Neurod1 and Foxa2 to maintain mature pancreatic beta-cell function. The EMBO Journal 34: 1417-1433. DOI: https://doi.org/10.15252/embj.201490819, PMID: 25828096

Jia Y, Zhao Y, Kusakizako T, Wang Y, Pan C, Zhang Y, Nureki O, Hattori M, Yan Z. 2020. TMC1 and TMC2 Proteins Are Pore-Forming Subunits of Mechanosensitive Ion Channels. Neuron 105: 310-321. DOI: https://doi.org/10. 1016/j.neuron.2019.10.017, PMID: 31761710

Jones JM, Montcouquiol M, Dabdoub A, Woods C, Kelley MW. 2006. Inhibitors of differentiation and DNA binding (lds) regulate Math1 and hair cell formation during the development of the organ of Corti. The Journal of Neuroscience 26: 550-558. DOI: https://doi.org/10.1523/JNEUROSCI.3859-05.2006, PMID: 16407553

Kelly MC, Chang Q, Pan A, Lin X, Chen P. 2012. Atoh1 directs the formation of sensory mosaics and induces cell proliferation in the postnatal mammalian cochlea in vivo. The Journal of Neuroscience 32: 6699-6710. DOI: https://doi.org/10.1523/JNEUROSCI.5420-11.2012, PMID: 22573692

Khimich D, Nouvian R, Pujol R, Tom Dieck S, Egner A, Gundelfinger ED, Moser T. 2005. Hair cell synaptic ribbons are essential for synchronous auditory signalling. Nature 434: 889-894. DOI: https://doi.org/10.1038/ nature03418, PMID: 15829963

Kolla L, Kelly MC, Mann ZF, Anaya-Rocha A, Ellis K, Lemons A, Palermo AT, So KS, Mays JC, Orvis J. 2020. Characterization of the development of the mouse cochlear epithelium at the single cell level. Nature Communications 11: 2389. DOI: https://doi.org/10.1038/s41467-020-16113-y, PMID: 32404924

Lee SI, Conrad T, Jones SM, Lagziel A, Starost MF, Belyantseva IA, Friedman TB, Morell RJ. 2013. A null mutation of mouse Kcna10 causes significant vestibular and mild hearing dysfunction. Hearing Research 300: 1-9. DOI: https://doi.org/10.1016/j.heares.2013.02.009, PMID: 23528307

Li Y, Liu H, Giffen KP, Chen L, Beisel KW, He DZZ. 2018a. Transcriptomes of cochlear inner and outer hair cells from adult mice. Scientific Data 5: 180199. DOI: https://doi.org/10.1038/sdata.2018.199

Li C, Shu Y, Wang G, Zhang H, Lu Y, Li X, Li G, Song L, Liu Z. 2018b. Characterizing a novel vGlut3-P2A-iCreER knockin mouse strain in cochlea. Hearing Research 364: 12-24. DOI: https://doi.org/10.1016/j.heares.2018.04. 006

Li X, Yu X, Chen X, Liu Z, Wang G, Li C, Wong EYM, Sham MH, Tang J, He J. 2019. Localization of TMC1 and LHFPL5 in auditory hair cells in neonatal and adult mice. FASEB Journal 33: 6838-6851. DOI: https://doi.org/ 10.1096/fj.201802155RR, PMID: 30808210

Li X, Bi Z, Sun Y, Li C, Li Y, Liu Z. 2020a. In vivo ectopic Ngn1 and Neurod1 convert neonatal cochlear glial cells into spiral ganglion neurons. FASEB Journal 34: 4764-4782. DOI: https://doi.org/10.1096/fj.201902118R

Li C, Li X, Bi Z, Sugino K, Wang G, Zhu T, Liu Z. 2020b. Comprehensive transcriptome analysis of cochlear spiral ganglion neurons at multiple ages. eLife 9: e50491. DOI: https://doi.org/10.7554/eLife.50491, PMID: 31913118

Liberman MC, Gao J, He DZ, Wu X, Jia S, Zuo J. 2002. Prestin is required for electromotility of the outer hair cell and for the cochlear amplifier. Nature 419: 300-304. DOI: https://doi.org/10.1038/nature01059, PMID: 12239568

Liu Z, Dearman JA, Cox BC, Walters BJ, Zhang L, Ayrault O, Zindy F, Gan L, Roussel MF, Zuo J. 2012a. Agedependent in vivo conversion of mouse cochlear pillar and Deiters' cells to immature hair cells by Atoh1 ectopic expression. The Journal of Neuroscience 32: 6600-6610. DOI: https://doi.org/10.1523/JNEUROSCI. 0818-12.2012 
Liu Z, Walters BJ, Owen T, Brimble MA, Steigelman KA, Zhang L, Mellado Lagarde MM, Valentine MB, Yu Y, Cox BC. 2012b. Regulation of p27Kip1 by Sox2 maintains quiescence of inner pillar cells in the murine auditory sensory epithelium. The Journal of Neuroscience 32: 10530-10540. DOI: https://doi.org/10.1523/JNEUROSCI. 0686-12.2012

Liu Z, Fang J, Dearman J, Zhang L, Zuo J. 2014a. In vivo generation of immature inner hair cells in neonatal mouse cochleae by ectopic atoh1 expression. PLOS ONE 9: e89377. DOI: https://doi.org/10.1371/journal. pone.0089377, PMID: 24586731

Liu H, Pecka JL, Zhang Q, Soukup GA, Beisel KW, He DZZ. 2014b. Characterization of transcriptomes of cochlear inner and outer hair cells. The Journal of Neuroscience 34: 11085-11095. DOI: https://doi.org/10.1523/ JNEUROSCI.1690-14.2014, PMID: 25122905

Lorenzen SM, Duggan A, Osipovich AB, Magnuson MA, Garcia-Anoveros J. 2015. Insm1 promotes neurogenic proliferation in delaminated otic progenitors. Mechanisms of Development 138: 233-245. DOI: https://doi.org/ 10.1016/j.mod.2015.11.001, PMID: 26545349

Matei V, Pauley S, Kaing S, Rowitch D, Beisel KW, Morris K, Feng F, Jones K, Lee J, Fritzsch B. 2005. Smaller inner ear sensory epithelia in Neurog 1 null mice are related to earlier hair cell cycle exit. Developmental Dynamics 234: 633-650. DOI: https://doi.org/10.1002/dvdy.20551, PMID: 16145671

McLean WJ, Yin X, Lu L, Lenz DR, McLean D, Langer R, Karp JM, Edge ASB. 2017. Clonal Expansion of Lgr5Positive Cells from Mammalian Cochlea and High-Purity Generation of Sensory Hair Cells. Cell Reports 18: 1917-1929. DOI: https://doi.org/10.1016/j.celrep.2017.01.066, PMID: 28228258

Mellado Lagarde MM, Cox BC, Fang J, Taylor R, Forge A, Zuo J. 2013. Selective ablation of pillar and deiters' cells severely affects cochlear postnatal development and hearing in mice. The Journal of Neuroscience 33: 1564-1576. DOI: https://doi.org/10.1523/JNEUROSCI.3088-12.2013, PMID: 23345230

Mellado Lagarde MM, Wan G, Zhang L, Gigliello AR, Mclnnis JJ, Zhang Y, Bergles D, Zuo J, Corfas G. 2014. Spontaneous regeneration of cochlear supporting cells after neonatal ablation ensures hearing in the adult mouse. PNAS 111: 16919-16924. DOI: https://doi.org/10.1073/pnas.1408064111, PMID: 25385613

Menendez L, Trecek T, Gopalakrishnan S, Tao L, Markowitz AL, Yu HV, Wang X, Llamas J, Huang C, Lee J. 2020. Generation of inner ear hair cells by direct lineage conversion of primary somatic cells. eLife 9: e55249. DOI: https://doi.org/10.7554/eLife.55249, PMID: 32602462

Montcouquiol M, Kelley MW. 2020. Development and Patterning of the Cochlea: From Convergent Extension to Planar Polarity. Cold Spring Harbor Perspectives in Medicine 10: a033266. DOI: https://doi.org/10.1101/ cshperspect.a033266, PMID: 30617059

Okumura H, Miyasaka Y, Morita Y, Nomura T, Mishima Y, Takahashi S, Kominami R. 2011. Bcl11b heterozygosity leads to age-related hearing loss and degeneration of outer hair cells of the mouse cochlea. Experimental Animals 60: 355-361. DOI: https://doi.org/10.1538/expanim.60.355, PMID: 21791875

Pan B, Geleoc GS, Asai Y, Horwitz GC, Kurima K, Ishikawa K, Kawashima Y, Griffith AJ, Holt JR. 2013. TMC1 and TMC2 are components of the mechanotransduction channel in hair cells of the mammalian inner ear. Neuron 79: 504-515. DOI: https://doi.org/10.1016/j.neuron.2013.06.019, PMID: 23871232

Parker A, Chessum L, Mburu P, Sanderson J, Bowl MR. 2016. Light and Electron Microscopy Methods for Examination of Cochlear Morphology in Mouse Models of Deafness. Current Protocols in Mouse Biology 6: 272-306. DOI: https://doi.org/10.1002/cpmo.10, PMID: 27584554

Ranum PT, Goodwin AT, Yoshimura H, Kolbe DL, Walls WD, Koh JY, He DZZ, Smith RJH. 2019. Insights into the Biology of Hearing and Deafness Revealed by Single-Cell RNA Sequencing. Cell Reports 26: 3160-3171. DOI: https://doi.org/10.1016/j.celrep.2019.02.053, PMID: 30865901

Ruel J, Emery S, Nouvian R, Bersot T, Amilhon B, Van Rybroek JM, Rebillard G, Lenoir M, Eybalin M, Delprat B. 2008. Impairment of SLC17A8 encoding vesicular glutamate transporter-3, VGLUT3, underlies nonsyndromic deafness DFNA25 and inner hair cell dysfunction in null mice. American Journal of Human Genetics 83: 278-292. DOI: https://doi.org/10.1016/j.ajhg.2008.07.008, PMID: 18674745

Scheffer DI, Shen J, Corey DP, Chen ZY. 2015. Gene Expression by Mouse Inner Ear Hair Cells during Development. The Journal of Neuroscience 35: 6366-6380. DOl: https://doi.org/10.1523/JNEUROSCI.512614.2015, PMID: 25904789

Seal RP, Akil O, Yi E, Weber CM, Grant L, Yoo J, Clause A, Kandler K, Noebels JL, Glowatzki E. 2008. Sensorineural deafness and seizures in mice lacking vesicular glutamate transporter 3. Neuron 57: 263-275. DOI: https://doi.org/10.1016/j.neuron.2007.11.032, PMID: 18215623

Shim K, Minowada G, Coling DE, Martin GR. 2005. Sprouty2, a mouse deafness gene, regulates cell fate decisions in the auditory sensory epithelium by antagonizing FGF signaling. Developmental Cell 8: 553-564. DOI: https://doi.org/10.1016/j.devcel.2005.02.009, PMID: 15809037

Shrestha BR, Chia C, Wu L, Kujawa SG, Liberman MC, Goodrich LV. 2018. Sensory Neuron Diversity in the Inner Ear Is Shaped by Activity. Cell 174: 1229-1246. DOI: https://doi.org/10.1016/j.cell.2018.07.007, PMID: 30078709

Shu Y, Li W, Huang M, Quan YZ, Scheffer D, Tian C, Tao Y, Liu X, Hochedlinger K, Indzhykulian AA. 2019. Renewed proliferation in adult mouse cochlea and regeneration of hair cells. Nature Communications 10: 5530. DOI: https://doi.org/10.1038/s41467-019-13157-7, PMID: 31797926

Simmons DD, Tong B, Schrader AD, Hornak AJ. 2010. Oncomodulin identifies different hair cell types in the mammalian inner ear. The Journal of Comparative Neurology 518: 3785-3802. DOI: https://doi.org/10.1002/ cne.22424, PMID: 20653034

Stone JS, Cotanche DA. 2007. Hair cell regeneration in the avian auditory epithelium. The International Journal of Developmental Biology 51: 633-647. DOI: https://doi.org/10.1387/ijdb.072408js, PMID: 17891722 
Tateya T, Sakamoto S, Ishidate F, Hirashima T, Imayoshi I, Kageyama R. 2019. Three-dimensional live imaging of Atoh1 reveals the dynamics of hair cell induction and organization in the developing cochlea. Development 146: dev177881. DOI: https://doi.org/10.1242/dev.177881, PMID: 31676552

Tong L, Strong MK, Kaur T, Juiz JM, Oesterle EC, Hume C, Warchol ME, Palmiter RD, Rubel EW. 2015. Selective deletion of cochlear hair cells causes rapid age-dependent changes in spiral ganglion and cochlear nucleus neurons. The Journal of Neuroscience 35: 7878-7891. DOI: https://doi.org/10.1523/JNEUROSCI.2179-14. 2015, PMID: 25995473

Tong B, Hornak AJ, Maison SF, Ohlemiller KK, Liberman MC, Simmons DD. 2016. Oncomodulin, an EF-Hand Ca2+ Buffer, Is Critical for Maintaining Cochlear Function in Mice. The Journal of Neuroscience 36: 1631-1635. DOI: https://doi.org/10.1523/JNEUROSCI.3311-15.2016, PMID: 26843644

Walters BJ, Coak E, Dearman J, Bailey G, Yamashita T, Kuo B, Zuo J. 2017. In Vivo Interplay between p27(Kip1), GATA3, ATOH1, and POU4F3 Converts Non-sensory Cells to Hair Cells in Adult Mice. Cell Reports 19: 307-320. DOI: https://doi.org/10.1016/j.celrep.2017.03.044, PMID: 28402854

Wang G, Li C, He S, Liu Z. 2021. Mosaic CRISPR-stop enables rapid phenotyping of nonsense mutations in essential genes. Development 148: dev196899. DOI: https://doi.org/10.1242/dev.196899, PMID: 33558388

Warchol ME, Corwin JT. 1996. Regenerative proliferation in organ cultures of the avian cochlea: identification of the initial progenitors and determination of the latency of the proliferative response. The Journal of Neuroscience 16: 5466-5477. DOI: https://doi.org/10.1523/JNEUROSCI.16-17-05466.1996, PMID: 8757259

Wiwatpanit T, Lorenzen SM, Cantu JA, Foo CZ, Hogan AK, Marquez F, Clancy JC, Schipma MJ, Cheatham MA, Duggan A. 2018. Trans-differentiation of outer hair cells into inner hair cells in the absence of INSM1. Nature 563: 691-695. DOI: https://doi.org/10.1038/s41586-018-0570-8, PMID: 30305733

Woods C, Montcouquiol M, Kelley MW. 2004. Math1 regulates development of the sensory epithelium in the mammalian cochlea. Nature Neuroscience 7: 1310-1318. DOI: https://doi.org/10.1038/nn1349, PMID: 15543141

Wu DK, Kelley MW. 2012. Molecular mechanisms of inner ear development. Cold Spring Harbor Perspectives in Biology 4: a008409. DOI: https://doi.org/10.1101/cshperspect.a008409, PMID: 22855724

Wu Z, Muller U. 2016. Molecular Identity of the Mechanotransduction Channel in Hair Cells: Not Quiet There Yet. The Journal of Neuroscience 36: 10927-10934. DOI: https://doi.org/10.1523/JNEUROSCI.1149-16.2016, PMID: 27798175

Xiong W, Grillet N, Elledge HM, Wagner TF, Zhao B, Johnson KR, Kazmierczak P, Muller U. 2012. TMHS is an integral component of the mechanotransduction machinery of cochlear hair cells. Cell 151: 1283-1295. DOl: https://doi.org/10.1016/j.cell.2012.10.041, PMID: 23217710

Yu HV, Tao L, Llamas J, Wang X, Nguyen JD, Trecek T, Segil N. 2021. POU4F3 pioneer activity enables ATOH1 to drive diverse mechanoreceptor differentiation through a feed-forward epigenetic mechanism. PNAS 118: e2105137118. DOI: https://doi.org/10.1073/pnas.2105137118, PMID: 34266958

Zhang H, Pan H, Zhou C, Wei Y, Ying W, Li S, Wang G, Li C, Ren Y, Li G. 2018. Simultaneous zygotic inactivation of multiple genes in mouse through CRISPR/Cas9-mediated base editing. Development 145: dev168906. DOI: https://doi.org/10.1242/dev.168906, PMID: 30275281

Zheng J, Shen W, He DZ, Long KB, Madison LD, Dallos P. 2000. Prestin is the motor protein of cochlear outer hair cells. Nature 405: 149-155. DOI: https://doi.org/10.1038/35012009, PMID: 10821263 